\title{
Correlation driven metallic and half-metallic phases in a band insulator
}

\author{
Soumen Bag $\odot,{ }^{1}$ Arti Garg $\odot,{ }^{2}$ and H. R. Krishnamurthy $\oplus^{1}$ \\ ${ }^{1}$ Department of Physics, Indian Institute of Science, Bangalore 560 012, India \\ ${ }^{2}$ Condensed Matter Physics Division, Saha Institute of Nuclear Physics, 1/AF Bidhannagar, Kolkata 700 064, India
}

(Received 14 February 2020; revised 26 March 2021; accepted 30 March 2021; published 16 April 2021)

\begin{abstract}
We demonstrate a mechanism for realizing ferrimagnetic metal, antiferromagnetic half-metal, and paramagnetic metal phases by tuning the onsite Coulomb repulsion $U$ in a band insulator. In a simple model of a correlated band insulator, namely the ionic Hubbard model at half-filling, and in the presence of a second neighbor hopping, we show that as $U$ is increased, first he insulating band gap is suppressed to zero at a critical $U_{1}$ and remains zero within the paramagnetic metallic phase. Interestingly, at a larger $U_{c}$, ferrimagnetic order turns on, leading to a ferrimagnetic metallic phase for $U_{c}<U<U_{2}$. For $U>U_{2}$, the system exhibits an antiferromagnetic half-metallic phase, followed finally by a transition into an antiferromagnetic Mott insulator. Our results, based on dynamical mean-field theory, suggest alternate routes for achieving magnetically ordered metallic and half-metallic phases which have potential applications in spintronics.
\end{abstract}

DOI: 10.1103/PhysRevB.103.155132

\section{INTRODUCTION}

Exciting new physics is known to emerge when $e$-e interactions are turned on in systems which are otherwise metallic [1]. For example, in the half-filled Hubbard model, strong correlations turn a metal into a Mott insulator, while weak to intermediate interactions result in insulating phases due to spin-density wave formation. In contrast, the fate of a band insulator in the presence of interactions has been investigated very little. In this paper, we demonstrate the surprising result that increasing the strength of $e$-e interactions in a simple model of a correlated band insulator can lead to a sequence of correlation-induced metallic phases: a paramagnetic metal first, a ferrimagnetic metal next, and then an antiferromagnetic half-metal, before the system finally ends up as an antiferromagnetic Mott insulator. Furthermore, known mechanisms for the realization of conducting phases in the vicinity of a Mott insulator require doping the system away from half-filling, as in the high- $T_{c}$ cuprates [1]. However, the mechanisms we present here for realizing metals and half-metals close to a Mott insulator are all at at half-filling, i.e., without doping, and the accompanying disorder.

Antiferromagnetic half-metals [2], where electrons of one spin orientation are in a metallic state while those of the opposite spin orientation are insulating, with no net magnetization, are of interest because of their outstanding potential in spintronics [3], but are yet to be realized experimentally. Routes for obtaining such phases are hence of importance. Most of the earlier theoretical suggestions, mainly using densityfunctional theory (DFT) [4], involve well-known correlated materials either with both local moments and itinerant electrons, with cation vacancies [5], or with hole doping [6]. The mechanism presented in this paper, where correlation drives a band insulator into metallic and half-metallic phases at halffilling, is thus distinct from all of these and also promises to lead to cleaner systems as no doping is required. Furthermore, most known ferrimagnets are insulating, while some doubleperovskites are likely ferrimagnetic but also half-metallic [7], so the ferrimagnetic metal phase we find is also exotic.

The model of a correlated band insulator (BI) we use is the ionic Hubbard model (IHM) on a square lattice at half-filling. The IHM is an extension [8-18] of the Hubbard model on any bipartite lattice, obtained by adding a staggered "ionic" potential $\Delta$. This doubles the unit cell, and additionally, when the Hubbard on-site interaction $U$ is zero, induces a gap between the two tight-binding electronic bands that result, making the system a BI at half-filling (corresponding to one electron per site).

For the half-filled IHM with only first neighbor hopping $t$ $(t-\mathrm{IHM})$ and with the system constrained to be paramagnetic, it is known that a Hubbard $U$ of order $\Delta$ leads to a closure of the gap in the single-particle spectrum, resulting in a correlation-induced paramagnetic metal (PM) phase sandwiched between (correlated) BI and Mott-insulator phases [19-22]. However, permitting antiferromagnetic (AF) order leads to a direct $\mathrm{BI} \rightarrow \mathrm{AF}$ Mott-insulator (AFI) transition preempting the formation of the PM phase [23-37]. Thus, the $t$-IHM at half-filling has only insulating phases in its phase diagram [38].

Here we show that adding a second neighbor hopping $t^{\prime}$ to the IHM ( $t-t^{\prime}$ IHM), which frustrates AF order as well as breaks particle-hole symmetry, not only restores the PM phase but also leads to the emergence of the exotic ferrimagnetic metal (FM) and AF half-metal (AFHM) phases mentioned above, over wide ranges of $U$ and $t^{\prime}$, as depicted in the rich phase diagram of Fig. 1.

The phases in Fig. 1 can be intuitively understood as follows (see also the discussion on simple limits of the IHM in the next section). For $U=0$, the $t-t^{\prime}$ IHM on the square lattice has two bands separated by an indirect band gap of 


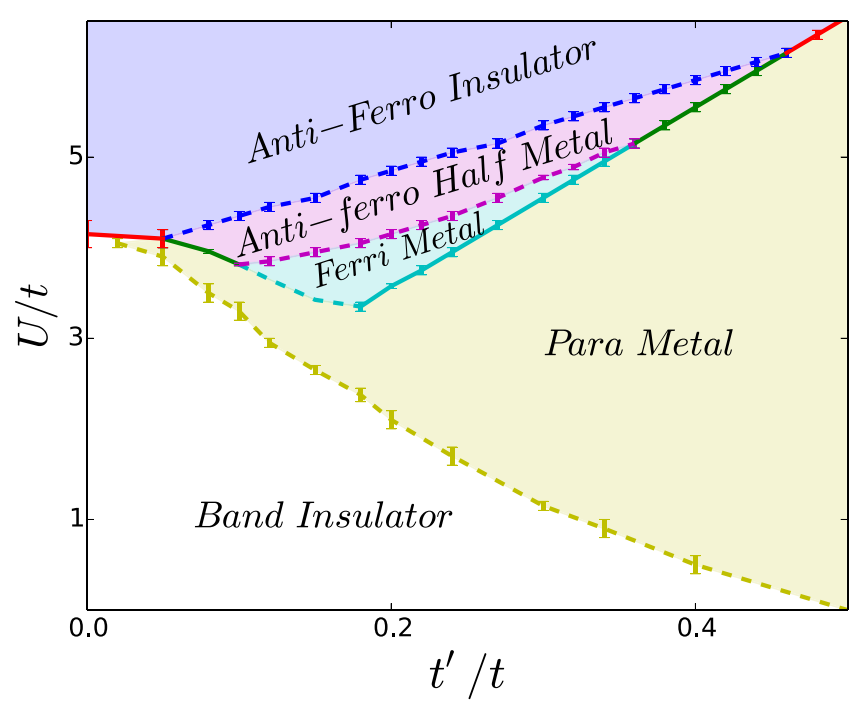

FIG. 1. Phase diagram of the $t-t^{\prime}$ IHM at half filling in the $t^{\prime}-U$ plane, obtained using the dynamical mean field theory (DMFT) combined with the continuous-time quantum Monte Carlo (CTQMC) impurity solver for $\beta t=50.0, \Delta=1.0 t$. Full (dashed) lines in the phase diagram indicate first-order (continuous) transition lines.

$2 \Delta-4 t^{\prime}$, with valence band maxima at the wave vectors $\mathbf{K}=(0, \pm \pi),( \pm \pi, 0)$ and conduction band minima at $\mathbf{K}^{\prime}=$ $( \pm \pi / 2, \pm \pi / 2$ ), leading to the BI phase at half-filling (for $t^{\prime}<\Delta / 2$ ). However, while $\Delta$ prefers doublons on one sublattice and holons on the other sublattice, $U$ and $t^{\prime}$ prefer single occupancies. Because of this competition, as $U$ increases, the effective quasiparticle band gap eventually closes, permitting gapless excitations. The resulting paramagnetic metal (PM) phase has particle-hole asymmetric electron and hole pockets (due to $t^{\prime}$ ), different occupancies of the two sublattices (due to $\Delta$ ), and nontrivial correlations (due to $U$ ). Hence, with further increase in $U$, and over a limited range of $t^{\prime}$, the $\mathrm{SU}(2)$ spin symmetry is spontaneously broken as magnetic order (induced by the $\mathrm{AF}$ exchange due to $U$ ) turns on, with opposite orientations but different magnitudes on the two sublattices. This leads to a phase with ferrimagnetic order and spin-polarized electron and hole pockets, i.e., the ferimagnetic metal (FM) phase. With continued increase in $U$, the quasiparticles with spins parallel to the magnetization on the sublattice with potential $+\Delta$ become gapped, resulting in a half-metallic phase, which, due to the half filling constraint, necessarily has $\mathrm{AF}$ order. This is the AF half-metal (AFHM) phase. Eventually, $U$ wins over $\Delta$ and $t^{\prime}$ and gaps open up in both the spin channels, resulting in the AFI phase [39].

The rest of this paper is organized as follows. In Sec. II, we present the model, its physics in simple limits and how this helps to qualitatively understand the phase diagram in Fig. 1, and the calculational methods we use for obtaining the results we present in this paper. In Sec. III, we present and discuss the central results of our paper in detail, including the different observables we calculate and how they lead to the phase diagram in Fig. 1. We end this paper with a concluding discussion in Sec. IV. The Appendixes contain technical details of the calculational methods used as well as some additional results not included in the main text.

\section{THE IHM HAMILTONIAN, SOME SIMPLE LIMITS, AND CALCULATIONAL METHODS}

The Hamiltonian for the $t-t^{\prime}$ IHM can be written using standard notation as

$$
\begin{aligned}
H_{\mathrm{IHM}}= & -t \sum_{\langle i j\rangle, \sigma}\left(\hat{c}_{j \sigma}^{\dagger} \hat{c}_{i \sigma}+\text { H.c. }\right)-t^{\prime} \sum_{\langle\langle i j\rangle\rangle, \sigma}\left(\hat{c}_{j \sigma}^{\dagger} \hat{c}_{i \sigma}\right) \\
& +\Delta \sum_{i \in A} \hat{n}_{i}-\Delta \sum_{i \in B} \hat{n}_{i}-\mu \sum_{i} \hat{n}_{i}+U \sum_{i} \hat{n}_{i \uparrow} \hat{n}_{i \downarrow} .
\end{aligned}
$$

Here $i$ labels the sites of a bipartite lattice (with two interpenetrating sublattices $A$ and $B$ ). $\hat{c}_{i \sigma}^{\dagger}$ creates an electron with spin $\sigma$ and $\hat{n}_{i}$ and $\hat{n}_{i \sigma}$ are the total and spin resolved number operators at the site $i$. As mentioned earlier, $t$ and $t^{\prime}$ are the amplitudes for first and second neighbor hopping, $U$ is the onsite Hubbard repulsion, and $\Delta$ is the staggered potential. The chemical potential $\mu$ is chosen so that the average site occupancy $\left(n_{A}+n_{B}\right) / 2=1$, corresponding to the "half-filling" constraint which we retain throughout this paper. Although all the results we present in our paper are specific to the two-dimensional square lattice, we expect the bulk of the physics we unearth to be generic to models with compact density of states in higher dimensions.

Two simple limits in which the $t-t^{\prime}$ IHM is exactly solvable and easy to understand are worth discussing.

For $U=0$, the noninteracting limit, the model is a oneparticle Hamiltonian that can be diagonalized exactly in terms of two bands with quasimomentum or wave-vector labels $\mathbf{k} \equiv$ $\left(k_{x}, k_{y}\right)$. The band dispersion relations in the full Brillouin zone (BZ) are given by

$$
\epsilon_{\mathbf{k}}^{ \pm}=-4 t^{\prime} \cos k_{x} \cos k_{y} \pm \sqrt{\Delta^{2}+\left(\gamma_{\mathbf{k}}\right)^{2}}
$$

with $\gamma_{\mathbf{k}} \equiv-2 t\left(\cos k_{x}+\cos k_{y}\right)$. When $t^{\prime}=0$, the two bands are separated in energy by a band gap $\left(E_{\text {gap }}\right)$ of $2 \Delta$ along the square contour corresponding to $k_{y}= \pm \pi \pm k_{x}$. So at half-filling, the lower band is completely filled and the upper band is empty, resulting in a band insulator (BI). When $t^{\prime} \neq 0$, the eigenstates (for either band) along these square contours are no longer degenerate. The bottom of the upper conduction band is $\epsilon_{\mathbf{K}^{\prime}}^{+}=\Delta$ at $\mathbf{K}^{\prime} \equiv( \pm \pi / 2, \pm \pi / 2)$, whereas the top of the lower valence band is $\epsilon_{\mathbf{K}}^{-}=4 t^{\prime}-\Delta$ at $\mathbf{K} \equiv$ $(0, \pm \pi),( \pm \pi, 0)$. Hence, for $t^{\prime} \neq 0$ and $\Delta>2 t^{\prime}$, the two bands have an indirect band gap $E_{\text {gap }}=2 \Delta-4 t^{\prime}$. As long as $E_{\text {gap }}>0$, the system (at half-filling) continues to be a band insulator (BI). As one increases $t^{\prime}$ from 0 , the band gap monotonically decreases from $2 \Delta$ and become zero at $t^{\prime}=\Delta / 2$, whence we get a BI to band-metal transition. It is to be expected that when $U$ is turned on starting from the BI phase, the system will retain the gap for single-particle excitations for values of the interaction $U$ much smaller than the gap. We continue to refer to the resulting phase as the BI phase, consistent with the phase diagram in Fig. 1. However, as we demonstrate in the next section, when $t^{\prime}$ is substantial the effective (renormalized) gap decreases continuously as $U$ increases, leading to the correlation induced transition from the BI to the PM phase, with the latter being adiabatically connected to the noninteracting band-metal. We also note that 
in the presence of $\Delta$ the system always has charge density wave order, with $n_{B}>n_{A}$.

Next, consider the "atomic" limit, corresponding to $t=$ $t^{\prime}=0$. When $U / 2<\Delta$, the ground state of the system has doublons (i.e., two electrons with opposite spins) on every B site and holons (i.e., no electrons) on every A site, corresponding to the maximal charge density wave order, and with a single-particle excitation gap equal to $\Delta-U / 2$. This is the atomic limit of the (correlated) BI phase. We can expect that turning on hopping with strengths smaller than the gap will leave the system in the same phase, and the resulting correlated BI phase is adiabatically connected to the weak-coupling BI phase discussed above. On the other hand, when $t=t^{\prime}=0$ and $U / 2>\Delta$, the ground state of the system is the (atomic limit of the) Mott insulator (MI) phase, with one electron at each site, and a gap for single-particle excitations equal to $U / 2-\Delta$. In this case, the system has free spin-1/2 local moments which can point in any direction and the ground state is $2^{N}$-fold degenerate, where $N$ is the number of sites in the lattice. The turning on of $t$ and $t^{\prime}$ induces first and second neighbor antiferromagnetic superexchange interactions [26], $J=4 t^{2} U /\left(U^{2}-4 \Delta^{2}\right)$ and $J^{\prime}=4 t^{\prime 2} / U$ respectively, leading to the antiferromagnetic (Mott) insulator (AFI) phase in Fig. 1. Finally, for $U=2 \Delta$ in the atomic limit, the system has gapless single-particle (electron or hole) excitations in addition to local moments. When the hopping is turned on and $|U-2 \Delta|$ is of order $t$ and $t^{\prime}$, one can expect exotic correlated metallic phases, with and without magnetism, as indeed confirmed by the phase diagram in Fig. 1. We re-emphasize that, as mentioned in the introduction and shown in this paper, these metallic phases arise only when $t^{\prime}$ is nonzero.

In order to reliably investigate and establish the realizability of the possibilities mentioned above, one needs a calculational method capable of handling the strong correlations involved. Our method of choice employed in this paper is the dynamical mean field theory (DMFT) [40,41], which includes all local (i.e., single-site) correlations exactly. The DMFT maps the IHM into two separate self-consistent impurity problems, one for each sublattice, to solve which we use the hybridization expansion continuous-time quantum Monte Carlo (CTQMC) [42] impurity solver, generally considered the best current solver for DMFT. For more details, see Appendix A.

The CTQMC mehod has the limitation that it provides data only for imaginary time or Matsubara frequencies (see the next section for more details). Hence, we complement the CTQMC results with results obtained using the iterated perturbation theory (IPT) impurity solver [43], known to be qualitatively and semiquantitatively consistent with the CTQMC and other methods [44] in the weak to intermediate interaction regimes. These results are also presented in the next section, while more details regarding the calculations are given in Appendix C.

Finally, we note that when $t^{\prime}$ is sizable, the correlationinduced BI to PM transition in Fig. 1 occurs at relatively small values of $U$, and is therefore accessible to a weak coupling approximation such as the Hatree-Fock mean field theory (HF-MFT). An unrestricted HFMFT also allows one in principle to access the order parameters characterizing the other phases in Fig. 1. Hence, we have carried out a detailed
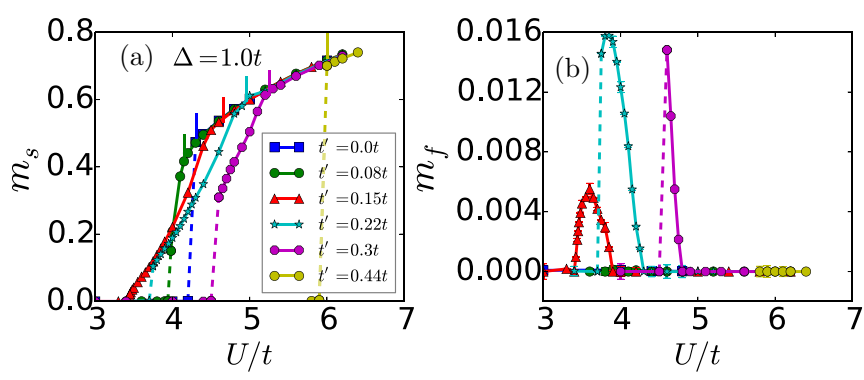

FIG. 2. The staggered magnetization $m_{s}$ and the uniform magnetization $m_{f}$, as functions of $U$ for different values of $t^{\prime}$ obtained from the DMFT +CTQMC calculations for $\beta t=50.0$ and $\Delta=1.0 t$. The $U$ dependence of $m_{s}$ (and of $m_{f}$ where appropriate) is in clear accordance with discontinuous, first-order jumps across the solid lines in Fig. 1 and continuous transitions across the dashed lines in Fig. 1.

investigation of the HF-MFT of the $t-t^{\prime}$ IHM, whose details and results are presented and discussed in Appendix D. We find that while HF-MFT yields all the same phases as in Fig. 1, and hence offers some insights into their nature, it grossly overestimates the stability of the magnetically ordered phases.

\section{RESULTS AND DISCUSSION}

In this section, we present and discuss our results [45] for the magnetic properties and the momentum distribution function, obtained using DMFT+CTQMC for fixed values of $\beta t \equiv t /\left(k_{B} T\right)=50.0$ and $\Delta=1.0 t$, and how they lead to the phase diagram in Fig. 1. We restrict our attention to positive values of $t^{\prime}$, as the physics for negative values of $t^{\prime}$ is obtainable simply via a particle-hole transformation. We also present results for real-frequency spectral functions obtained using DMFT + IPT.

\section{A. Staggered and uniform magnetization}

Figure 2 shows our results for the staggered magnetization $m_{s} \equiv\left(m_{z A}-m_{z B}\right) / 2$ and the uniform magnetization $m_{f} \equiv$ $\left(m_{z A}+m_{z B}\right) / 2$, where $m_{z \alpha} \equiv n_{\alpha \uparrow}-n_{\alpha \downarrow}$ denotes the magnetization for sublattice $\alpha$, as functions of $U / t$ for various values of $t^{\prime}$. For every $t^{\prime}$, magnetic order sets in above a threshold $U_{c}\left(t^{\prime}\right)$ of $U$. For $0.12 t<t^{\prime}<0.36 t$, both $m_{f}$ and $m_{s}$ turn on at the transition and are nonzero in a range $U_{c}\left(t^{\prime}\right)<U<U_{2}\left(t^{\prime}\right)$ (although the $m_{f}$ values are small), and the system has ferrimagnetic order. As $U \rightarrow U_{2}\left(t^{\prime}\right), m_{f} \rightarrow 0$, and for larger $U$ the system has AF order. For $t^{\prime}$ outside the above range, $m_{f}$ is zero for all values of $U$ and the system goes from paramagnetic to AF phases via first-order transitions across $U_{c}\left(t^{\prime}\right)$.

The threshold $U_{c}\left(t^{\prime}\right)$ has a nonmonotonic variation as $t^{\prime}$ increases, first decreasing and reaching a minimum around $t^{\prime}=0.18 t$ and then increasing again. This arises from two competing aspects, namely the size of the band gap and the degree of magnetic frustration. As mentioned earlier, the noninteracting $t-t^{\prime}$ IHM has a band gap $\left(2 \Delta-4 t^{\prime}\right)$ that decreases as $t^{\prime}$ increases, hence the initial decrease in $U_{c}$. But increasing $t^{\prime}$ also causes increasing frustration of the AF exchange interactions, hence the increase in $U_{c}$ for larger $t^{\prime}$. Furthermore, the jump in $m_{s}$ at the first-order AF transition for 
small $t^{\prime}$ decreases to zero as $t^{\prime} \rightarrow .12 t$, and the ferrimagnetic transition for $0.12 t<t^{\prime}<0.18 t$ is continuous, with both $m_{s}$ and $m_{f}$ turning on smoothly at $U_{c}$. For $t^{\prime}>0.18 t$, both magnetizations again turn on with a jump, and the transition becomes first order again, though the width in $U$ of the FM phase and the jump in $m_{f}$ at $U_{c}$ go to zero as $t^{\prime} \rightarrow 0.36 t$.

As discussed in greater detail in Appendix B, because of the presence of the staggered potential the spin-resolved staggered densities $\delta n_{\sigma} \equiv\left(n_{B \sigma}-n_{A \sigma}\right) / 2$ are finite for all $t^{\prime}, U$. They decrease as $U$ increases, as empty or doubly occupied sites are disfavored because of $U$ and the half-filling constraint, are spin symmetric (spin asymmetric) in the paramagnetic (magnetically ordered) phases, and show discontinuities (kinks) across first-order (continuous) phase transitions.

\section{B. Momentum distribution function}

Whether a system is metallic or insulating is ideally characterized by whether the zero-temperature single-particle spectral function or density of states (DOS), $\rho(\omega)$, is finite or zero at the Fermi level $(\omega=0)$. With the CTQMC method, one can only obtain the Green's function at the Matsubara frequencies, $G\left(i \omega_{n}\right)$ with $\omega_{n} \equiv(2 n+1) \pi / \beta$. Since $\rho(\omega)=$ $-\pi^{-1} \operatorname{Im} G\left(\omega^{+}\right)$, an analytic continuation of $G\left(i \omega_{n}\right)$ to real frequencies is required for obtaining the DOS, which is a difficult step with many subtle issues [46]. As an alternative procedure that avoids analytic continuation altogether, we use the momentum distribution functions (MDF) (see Appendix B for more details), $n_{\alpha, \mathbf{k}, \sigma}=\frac{1}{\beta} \sum_{n} G_{\alpha, \sigma}\left(\mathbf{k}, i \omega_{n}\right)+\frac{1}{2}(\alpha=A, B$ is the sublattice index) to distinguish whether the system is metallic or insulating. The MDF can be directly measured in cold-atom experiments [47] and can also be obtained from integrated spectral functions which are measured routinely using ARPES [48].

In the correlated BI phase at half-filling, which is adiabatically connected with the noninteracting BI phase where $\mu$ lies within the insulating gap, $n_{\mathbf{k}, \sigma} \equiv \frac{1}{2} \sum_{\alpha} n_{\alpha, \mathbf{k}, \sigma}$ has a constant value of $\frac{1}{2}$ throughout the Brillouin zone (BZ). In the PM state, adiabatically connected to the state in which both the noninteracting bands cross the Fermi level, we get (interaction-renormalized) electron pockets in the conduction band, with $n_{\mathbf{k}, \sigma}$ close to but less than 1.0, and hole pockets in the valence band, with small values of $n_{\mathbf{k}, \sigma}$, in such a way as to ensure half-filling. One can similarly use the spin dependence of the momentum distribution functions combined with the information on the magnetic order parameters to distinguish the FM and AFHM phases, as discussed below.

Figure 3 depicts $n_{\mathbf{k}, \sigma}$ over the full BZ for $t^{\prime}=0.2 t$ for three values of $U$ that lie respectively in the PM, FM, and AFHM regions of the phase diagram in Fig. 1. For $U=3.4 t, n_{\mathbf{k}, \sigma}$ is spin symmetric and has clearly visible (renormalized) electron pockets [peaks at $\left.\mathbf{k}=\mathbf{K}^{\prime} \equiv( \pm \pi / 2, \pm \pi / 2)\right]$ and hole pockets [dips at $\mathbf{k}=\mathbf{K} \equiv(0, \pm \pi),( \pm \pi, 0)]$, corresponding to the PM phase. As $U$ is increased and magnetic order sets in, $n_{\mathbf{k}, \sigma}$ (top panel of Fig. 3, for $U=3.8 t$ ) develops spin asymmetry, with the $\uparrow$ spin channel having bigger electron and hole pockets and also a larger net occupancy compared to the $\downarrow$ spin channel, corresponding to the FM phase. On further increasing $U$ (bottom panel of Fig. 3, for $U=4.6 t$ ), only $n_{\mathbf{k}, \uparrow}$ has Fermi pockets while $n_{\mathbf{k}, \downarrow}=\frac{1}{2}$ everywhere in the BZ. Thus, the $\downarrow$

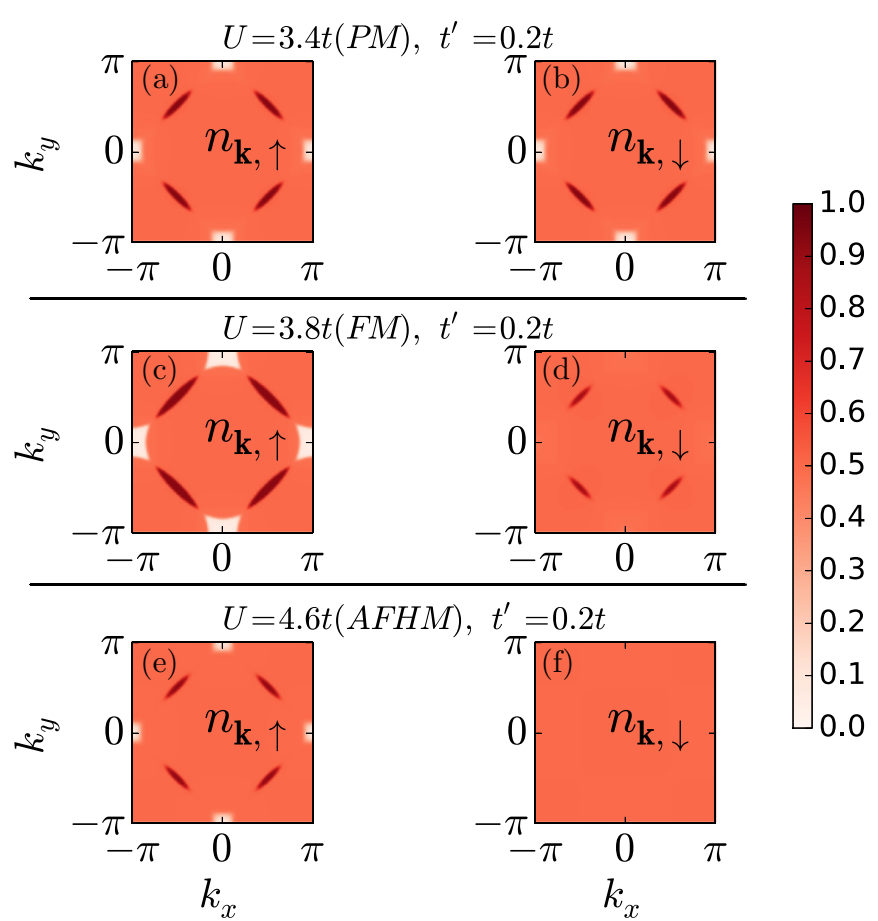

FIG. 3. False color plots of $n_{\mathbf{k}, \sigma}$ over the full BZ obtained using DMFT +CTQMC, for $\beta t=50.0, \Delta=1.0 t$, and $t^{\prime}=0.2$ for three different values of $U$. Top panels: PM phase, with spin symmetric electron and hole pockets. Middle panels: FM phase, with electron and hole pockets for the up spin but much smaller electron pockets and almost no hole pockets for the down spin. Bottom panels: AFHM phase, with only up-spin electron and hole pockets. For the down-spin electrons, $n_{\mathbf{k}, \downarrow}=0.5$ over the entire BZ, showing their insulating character.

spin channel is insulating with a finite band gap while the $\uparrow$ spin channel is a metal with both conduction and valence bands crossing the Fermi level, while the net occupancy in each channel is the same due to the half-filling constraint, corresponding to the AFHM phase. A different depiction of the same data is shown in Fig. 6 in Appendix B.

Figure 4 shows $n_{e \sigma} \equiv\left(n_{\mathbf{K}^{\prime} \sigma}-\frac{1}{2}\right)$ and $n_{h \sigma} \equiv\left(n_{\mathbf{K} \sigma}-\frac{1}{2}\right)$ as functions of $U$ for several representative values of $t^{\prime}$, chosen to show the different sequences of phases and phase transitions that occur in the half-filled $t-t^{\prime}$ IHM. For example, consider the panel for $t^{\prime}=0.2 t$, for which all the five phases that we have discussed exist for different ranges of $U$ (see Fig. 1). In the insulating phases, $n_{e}$ and $n_{h}$ are zero for both spin orientations, and the onset of metallicity is indicated by them deviating from zero. The first transition is continuous and from the BI to the PM phase, with spin symmetric deviations, across the blue curve in the phase diagram of Fig. 1. The second transition is into the FM phase, where $n_{e}$ and $n_{h}$ are still nonzero for both $\sigma$ values, but there is a spin asymmetry. The third transition is from the FM to the AFHM, where $n_{e}$ and $n_{h}$ differ from zero for only one spin channel, whereas the other spin channel is insulating. The final transition at large $U$ is a first-order transition to the AFI phase, with $n_{e}$ and $n_{h}$ becoming zero for both the spin channels. Similarly, the trends of $n_{e}$ and $n_{h}$ shown in the other panels (see also Fig. 7 in Appendix B) are consistent with Fig. 1. 

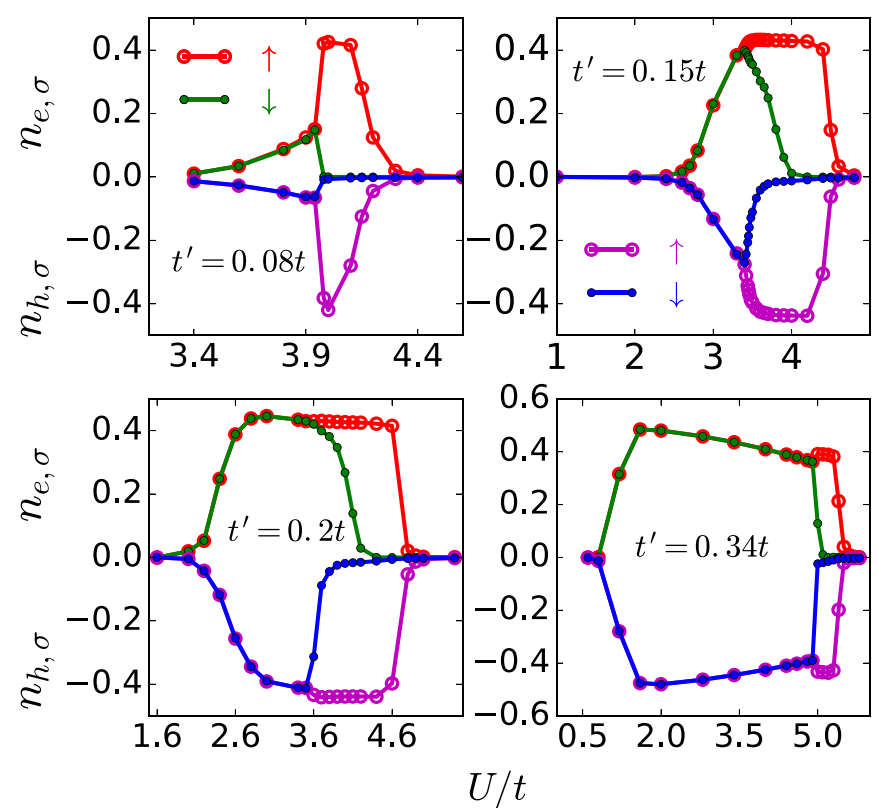

FIG. 4. $n_{e \sigma}$ (red and green curves) and $n_{h \sigma}$ (magenta and blue curves) vs $U$ for four different values of $t^{\prime}$. In the metallic phases, $n_{e \sigma}>0$ indicates electron pockets and $n_{h \sigma}<0$ indicates hole pockets. For $t^{\prime}=0.2$, as $U / t$ increases, there is a BI to PM transition, followed by a transition into the FM phase where $n_{e \sigma}>0$ for both the spin channels while only $n_{h \uparrow} \neq 0$. This is followed by a transition into an AFHM phase, where only $n_{e \uparrow}$ and $n_{h \uparrow}$ are nonzero. For large $U / t$, the AFI phase prevails, with $n_{e \sigma}=0=n_{h \sigma}$ for both spin channels. Other panels similarly show the phases for other values of $t^{\prime}$ and also how the ranges in $U$ over which the different phases exist change with $t^{\prime}$, consistent with Fig. 1.

\section{Results from DMFT +IPT}

We note that CTQMC calculations are possible only at finite temperatures; therefore, even at the lowest possible temperatures accessible to us numerically, metallic and insulating phases are clearly distinguishable only if the insulating gap is much larger than $k_{B} T$. Close to the metal-insulator transition point, where the gap becomes small, numerically it becomes hard to locate the transition, as it is really a crossover. Therefore, as mentioned earlier, we have also studied the $t-t^{\prime}$ IHM using iterative perturbation theory (IPT) as the impurity solver for the DMFT [40,43], where we have the advantage of being able to calculate the zero-temperature Green's function at real frequencies; see Appendix C for details.

All the phases of the $t-t^{\prime}$ IHM found in our DMFT + CTQMC calculations are also observed within the DMFT+IPT. Figure 5 presents the spin-resolved, sublattice-averaged, single-particle DOS $\rho_{\sigma}(\omega)$ obtained within DMFT + IPT for $t^{\prime}=0.3 t$ and $\Delta=1.0 t$ for several values of $U / t$. It shows that, consistent with the phase diagram in Fig. 1, the system indeed evolves in the sequence $\mathrm{BI} \rightarrow \mathrm{PM} \rightarrow \mathrm{FM} \rightarrow \mathrm{AFHM} \rightarrow \mathrm{AFI}$ as $U / t$ increases (DOS for the BI phase, with spin-symmetric gaps at the Fermi level, not shown). This reaffirms the reliability of our analysis based on the momentum distribution function within DMFT +CTQMC and the presence of the correlation-induced PM, FM, and AFHM phases in the $t-t^{\prime}$ IHM, which is one
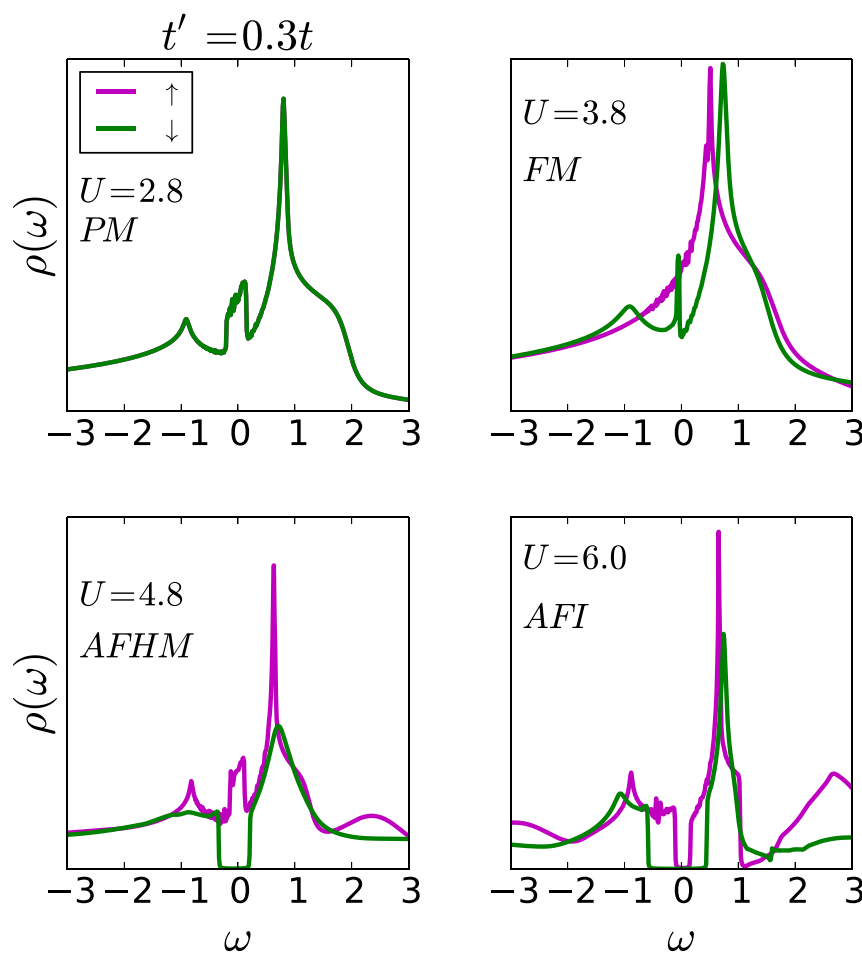

FIG. 5. Single-particle density of states $\rho_{\sigma}(\omega)$ obtained within DMFT + IPT for $t^{\prime}=0.3 t$ and $\Delta=1.0 t$ shown for several values of $U / t$. As $U / t$ increases starting from a PM phase, the system first becomes a FM, with $\rho_{\uparrow}(\omega) \neq \rho_{\downarrow}(\omega)$ and the DOS in the occupied part of the spectrum being smaller for down-spin compared to up-spin, resulting in ferrimagnetic order. As $U / t$ increases further, the down-spin channel gets gapped, resulting in the AFHM phase. Eventually, a gap opens up in the up-spin channel as well, and the system becomes an AFI.

of the main achievements of this work. Needless to say, the locations of the phase transition lines will be different in DMFT +CTQMC compared to DMFT + IPT, and we expect the former to be more accurate. Nevertheless, the consistency between the results from the two very different methods is encouraging and suggests that the DMFT +IPT can be used to obtain reliable results for other properties, such as transport, which are difficult to calculate using the DMFT +CTQMC method because of the limitations connected with analytic continuation discussed above. We hope to carry out such studies in the future.

\section{CONCLUDING COMMENTS}

In conclusion, in this paper we have demonstrated in a simple model of a correlated band insulator at half-filling that correlations can induce AF half-metal, ferrimagnetic metal, and paramagnetic metallic phases over broad parameter regimes. Our proposal for realizing the AFHM phase is distinct from the proposals mentioned in the introduction [2-6] as well as a recent model-based proposal [49] which also involves doping a parent system. The AFHM phase in our work is realized at half-filling, thereby avoiding the disorder that accompanies doping. Our mechanism should be realizable in band-insulating materials with two inequivalent strongly 
correlated sites per unit cell, frustration in hopping and antiferromagnetic exchange, and lack of particle-hole symmetry.

The question of a whether a stable correlation-induced metallic phase can intervene between the BI and the AFI phases in the half-filled IHM has been around for more than a decade. Our work presents a definitive answer, in the affirmative, by analyzing the $t-t^{\prime}$ IHM at half-filling and furthermore has uncovered a rich set of additional phases. In addition to demonstrating a scenario where conducting phases arise in the close vicinity of a Mott insulator without any doping, our work also implements a method for identifying metallic and insulating phases based on the momentum-distribution function obtained from CTQMC calculations without the need of analytic continuation.

It will certainly be interesting to investigate the existence of these correlation-induced metallic and half-metallic phases as well as the nature of the various phase transitions involved further, theoretically, using the more sophisticated and computationally demanding quantum cluster and other methods [50-52], and experimentally, in cold atom systems and real materials. The IHM has been simulated recently in ultracold atom experiments [53]. Recent developments in layered materials and heterostructures also generate many contexts where the qualitative physics can be understood in terms of the IHM. For example, bilayers of 2-d materials, e.g., bilayer graphene, in the presence of a transverse electric field which induces a potential difference between the two layers, can be modeled in terms of an IHM [54]. We note also that the IHM can arise as an emergent effective model when spontaneous symmetry breaking leads to the development of a commensurate two-sublattice charge density wave induced by electron-phonon or first neighbor Coulomb interactions. We hope that our work will motivate further theoretical as well as experimental research looking for the correlation-induced paramagnetic metal, ferrimagnetic metal, and antiferromagnetic half-metal phases in these systems and contexts. Additionally [45], the nature of the phases and phase transitions in 2-d BI systems with strong correlations, where magnetic long-range order is suppressed at finite temperatures due to long wavelength fluctuations (unless there is spin anisotropy), poses fascinating and challenging problems. We hope that our work presented here will stimulate further research in these directions.

\section{ACKNOWLEDGMENTS}

A.G. and H.R.K. gratefully thank the Department of Science and Technology, Ministry of Science and Technology, India, and its Science and Engineering Research Board, for financial support under Grants No. CRG/2018/003269 and No. $\mathrm{SB} / \mathrm{DF} / 005 / 2017$, respectively. We also thank J. K. Freericks for valuable feedback regarding early versions of this paper.

\section{APPENDIX A: DETAILS OF DMFT + CTQMC METHOD}

The formulation of the dynamical mean-field theory (DMFT) for the IHM and the use of CTQMC as the impurity solver have been discussed in detail in Sec. II of our earlier work [26] (corresponding to $t^{\prime}=0$ ) and in the references cited therein. Here we confine ourselves to drawing attention to the specific differences that arise due to the presence of a nonzero $t^{\prime}$.

Even in the presence of $t^{\prime}$, the expression for the $\mathbf{k}$ and spin-dependent $(2 \times 2)$ matrix Green's function for the IHM in terms of the local spin-dependent self-energies continues to be of the form given by Eq. (2) of Ref. [26], i.e.,

$$
\left[\mathbf{G}^{\sigma}\left(\mathbf{k}, i \omega_{n}\right)\right]_{\alpha \beta}=\left(\begin{array}{cc}
\zeta_{A \sigma}\left(\mathbf{k}, i \omega_{n}\right) & -\gamma_{\mathbf{k}} \\
-\gamma_{\mathbf{k}} & \zeta_{B \sigma}\left(\mathbf{k}, i \omega_{n}\right)
\end{array}\right)^{-1}
$$

where the two-valued matrix indices $\alpha$ and $\beta$ correspond to the two sublattice indices (A, B). However, the diagonal entries on the right-hand side are now $\mathbf{k}$ dependent, due to the intrasublattice hopping induced by $t^{\prime}$, where as the off-diagonal terms, due to the intersublattice hopping $t$, are given by $-\gamma_{\mathbf{k}}=2 t\left(\cos k_{x}+\cos k_{y}\right)$ as before. For the diagonal terms, we now have

$$
\zeta_{\alpha \sigma}\left(\mathbf{k}, i \omega_{n}\right)=\zeta_{\alpha \sigma}\left(i \omega_{n}\right)-\gamma_{\mathbf{k}}^{\prime}
$$

Here, $\alpha=A, B$ is the sublattice index,

$$
\begin{aligned}
& \zeta_{A \sigma}\left(i \omega_{n}\right)=i \omega_{n}+\mu-\Delta-\Sigma_{A, \sigma}\left(i \omega_{n}\right), \\
& \zeta_{B \sigma}\left(i \omega_{n}\right)=i \omega_{n}+\mu+\Delta-\Sigma_{B, \sigma}\left(i \omega_{n}\right),
\end{aligned}
$$

in terms of the two local self-energies $\Sigma_{\alpha, \sigma}\left(i \omega_{n}\right)$ of the two separate self-consistent impurity problems on sites A and B as before, and $\gamma_{\mathbf{k}}^{\prime}=-4 t^{\prime} \cos k_{x} \cos k_{y}$.

It is straightforward to verify that the diagonal components of the $\mathbf{k}$ and spin-dependent matrix Green's function are hence given by

$$
G_{\alpha \sigma}\left(\mathbf{k}, i \omega_{n}\right)=\frac{\zeta_{\bar{\alpha} \sigma}\left(i \omega_{n}\right)-\gamma_{\mathbf{k}}^{\prime}}{\left[\zeta_{A \sigma}\left(i \omega_{n}\right)-\gamma_{\mathbf{k}}^{\prime}\right]\left[\zeta_{B \sigma}\left(i \omega_{n}\right)-\gamma_{\mathbf{k}}^{\prime}\right]-\gamma_{\mathbf{k}}^{2}},
$$

where $\bar{\alpha}=B, A$ for $\alpha=A, B$. The local Green's functions on an $A$ or $B$ site are obtained by performing the $\mathbf{k}$ sum,

$$
G_{\alpha \sigma}\left(i \omega_{n}\right)=\frac{1}{N} \sum_{\mathbf{k}} G_{\alpha \sigma}\left(\mathbf{k}, i \omega_{n}\right)
$$

where $N$ is the total number of $\mathbf{k}$ points in the square Brillouin zone (BZ). Unlike in the model with $t^{\prime}=0$, the sum cannot be easily converted into an integral over a density of states as it involves two different energy dispersions. We carry it out numerically, using a $\left(2 L_{g} \times 2 L_{g}\right)$ grid over the square BZ, with the crystal momentum components $k_{x}$ and $k_{y}$ defined as $\frac{\left(i-L_{g}\right) \pi}{L_{g}}+\frac{\pi}{2 L_{g}}$ where $i$ runs from 0 to $\left(2 L_{g}-1\right)$. Because of the symmetry of Hamiltonian on the square lattice BZ, one does not need to sample the whole BZ; sampling a 1/8 wedge of the BZ with appropriate weights will do the job. Momentum sums are carried out over these triangular wedges. (The triangular regime is bordered by $k_{x}=k_{y}$ where $-\pi<k_{x}<0$. $\mathbf{k}$ points on the $k_{x}=k_{y}$ line are ascribed weights of 4 and all other points in the triangle carry weights of 8.) Based on our experience with the HF-MFT calculations (see Appendix D), where we find that calculations made using $L_{g}=1000$ yield the same results as those with the larger $L_{g}=2000$ and 4000; all the data reported in this paper have been obtained using $L_{g}=1000$.

The hybridization functions on the A and B sites, which are the inputs to the CTQMC impurity solver, are then 
obtained as

$$
D_{\alpha, \sigma}\left(i \omega_{n}\right)=\zeta_{\alpha \sigma}\left(i \omega_{n}\right)-\left[G_{\alpha, \sigma}\left(i \omega_{n}\right)\right]^{-1} .
$$

The CTQMC code (we use the CT-HYB package by Kristjan Haule [55] that has been graciously made available in the public domain) then generates the local self-energies on the $\mathrm{A}$ and $\mathrm{B}$ sites as functions of the Matsubara frequencies. Starting from a trial self-energy, e.g., the HartreeFock self-energies, the computation of the local Green's functions, the hybridization functions, and the local selfenergies are then iterated to convergence. Even if we start from a trial self-energy that corresponds to a paramagnetic state, the CTQMC code we use is sophisticated enough to self-consistently generate nonzero values for the local magnetizations, $m_{A}$ and $m_{B}$, as appropriate. The convergence criteria we used were that $n_{A}$ and $n_{B}$ were converged to an accuracy of 0.0002 , and the updating of chemical potential was stopped once $\left|\left(n_{A}+n_{B}\right)-2.0\right|$ reached 0.001. Our CTQMC calculations for the self-energy typically involved $10^{7}$ Monte Carlo steps. For most of the parameter regimes we studied, around 150 self-consistent DMFT cycles were required for convergence, whereas for parameters near phase transition lines it took around 200 cycles.

The momentum distribution function $n_{\alpha, \mathbf{k}, \sigma}$ is calculable in a straightforward way using the converged self-energies in the expression for the $\mathbf{k}$, sublattice, and spin-resolved diagonal components of the matrix Green's function given above by performing the Matsubara frequency sum

$$
n_{\alpha, \mathbf{k}, \sigma}=\frac{1}{\beta} \sum_{i \omega_{n}} G_{\alpha, \sigma}\left(\mathbf{k}, i \omega_{n}\right)+\frac{1}{2}
$$

as discussed in the main text. The Fermi surface is defined by the locus of points in the BZ where there is a jump in the momentum distribution function. These points can be located by determining where the gradient with respect to $\mathbf{k}$ of $n_{\alpha, \mathbf{k}, \sigma}$ has the maximum magnitude. However, visually they are fairly easy to see in false color plots of the momentum distribution functions, as discussed in the main text.

The various phase transition points in the phase diagram of the main text were identified using the following criteria. Needless to say, the parts of the $U_{c}$ line corresponding to the first-order magnetic transitions were easy to identify from the staggered magnetization data. The part of the $U_{c}$ line corresponding to the $\mathrm{PM} \rightarrow \mathrm{FM}$ continuous transition was inferred by checking for $m_{s}$ and $m_{f}$ exceeding 0.001 . From the $n_{\mathbf{k}}$ data, a transition from the AFHM phase to the AFI phase was inferred when $n_{\mathbf{k}}$ differed from 0.5 by less than 0.02 . The BI to metal transition was inferred when $n_{\mathbf{k}}$ differed from 0.5 by more than 0.02 .

\section{APPENDIX B: MOMENTUM DISTRIBUTION FUNCTION AND STAGGERED DENSITY WITHIN DMFT +CTQMC}

Here we present some additional results from our momentum distribution and staggered density calculations using DMFT +CTQMC that are complementary to the results presented and discussed in the main text.

Figure 6 shows the numerical values of $\left(n_{\mathbf{k}, \sigma}-0.5\right)$ on paths along high-symmetry directions in the $\mathrm{BZ}$ for the $t-t^{\prime}$
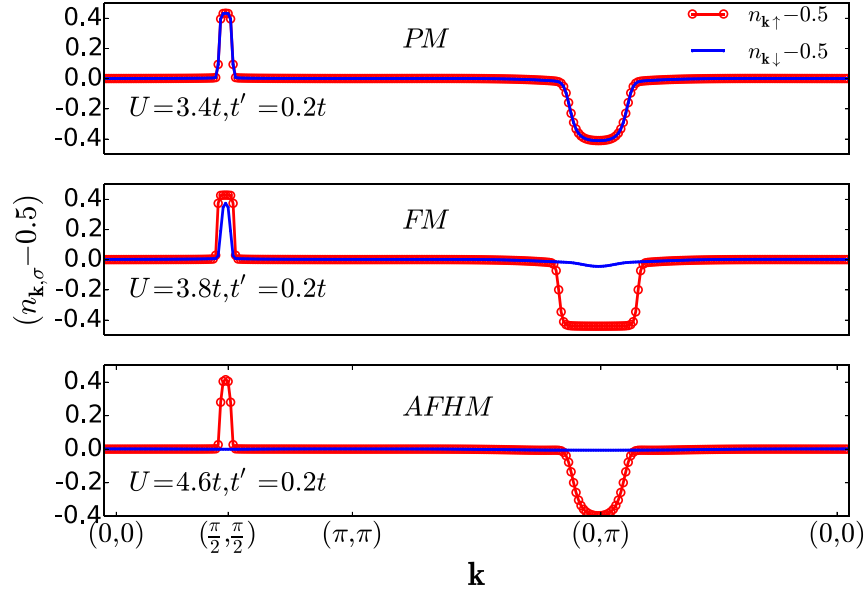

FIG. 6. Numerical values of $\left(n_{\mathbf{k}, \sigma}-0.5\right)$ on paths along highsymmetry directions in the BZ for the $t-t^{\prime}$ IHM at half-filling obtained using DMFT +CTQMC for $\beta t=50.0, \Delta=1.0 t$, and $t^{\prime}=$ $0.2 t$, for the same $U / t$ values of 3.4, 3.8, and 4.6 as in Fig. 3 of the main text, chosen to correspond respectively to the PM, FM, and AFHM phases. Top panel: PM phase, with spin symmetric electron pockets around the symmetry points $( \pm \pi / 2, \pm \pi / 2)$, where $n_{\mathbf{k}, \sigma}$ is close to 1 , and hole pockets around the symmetry points $( \pm \pi, 0)$ and $(0, \pm \pi)$, where $n_{\mathbf{k}, \sigma}$ is close to zero. Middle panel: FM phase, with electron and hole pockets for the up spin, but much smaller electron pockets and almost no hole pockets for the down spin. Bottom panel: AFHM phase, with only up-spin electron and hole pockets. For the down-spin electrons, $n_{\mathbf{k}, \downarrow}=0.5$ on all the paths, showing their insulating character.

IHM at half-filling obtained using DMFT + CTQMC for $\beta t=$ 50.0, $\Delta=1.0 t$, and $t^{\prime}=0.2 t$, for the same $U / t$ values of 3.4, 3.8, and 4.6 as in Fig. 3 of the main text, chosen to correspond respectively to the PM, FM, and AFHM phases. In the top panel, corresponding to the PM phase, the momentum distribution function is spin symmetric and has clearly visible renormalized electron pockets with $n_{\mathbf{k}, \sigma}$ close to 1 at $\mathbf{k}=( \pm \pi / 2, \pm \pi / 2)$ and hole pockets with $n_{\mathbf{k}, \sigma}$ close to 0 at $\mathbf{k}=(0, \pm \pi),( \pm \pi, 0)$. Note also the particle-hole asymmetry because of the presence of $t^{\prime}$. In the middle panel, corresponding to the FM phase, the electron and hole pockets for the up-spin electrons are slightly larger, but for the down-spin electrons the electron pockets are smaller, and there are almost no hole pockets. In the bottom panel, corresponding to the AFHM phase, there are only up-spin electron and hole pockets. For the down-spin electrons, $n_{\mathbf{k}, \downarrow}=0.5$ on all the paths, showing their insulating character.

Figure 7 shows $n_{e \sigma} \equiv\left(n_{\mathbf{K}^{\prime} \sigma}-\frac{1}{2}\right)$ and $n_{h \sigma} \equiv\left(n_{\mathbf{K} \sigma}-\frac{1}{2}\right)$ at $\mathbf{K}^{\prime}=(\pi / 2, \pi / 2)$ (red and green curves) and $\mathbf{K}=(\pi, 0)$ (magenta and blue curves) as a function of $U$ for several values of $t^{\prime}$ complementary to the values for which the same quantities were shown in Fig. 4 of the main text. We recall that in the insulating phases these are both zero and the onset of metallicity is indicated by their deviating from zero. Thus, the panel for $t^{\prime}=0.0 t$ shows that the system is always insulating, whereas the panel for $t^{\prime}=0.05 t$ corresponds to the first value of $t^{\prime}$ where the PM phase makes an appearance in our calculations. The panel for $t^{\prime}=0.4 t$ illustrates the shrinking of the range of $U$ corresponding to the AFHM phase with increasing $t^{\prime}$ (see 

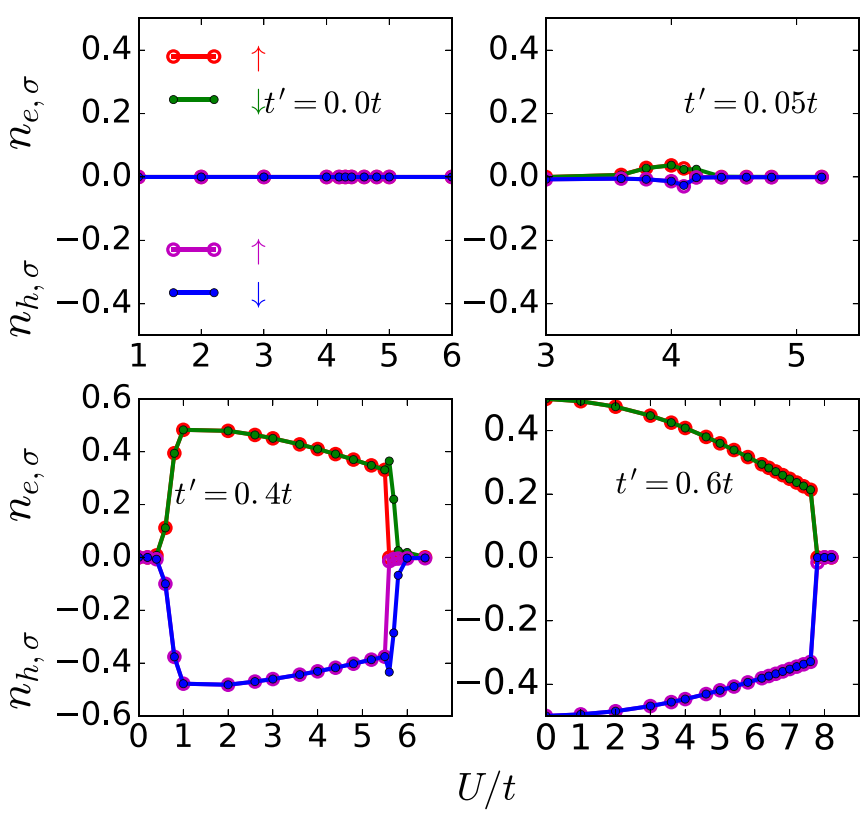

FIG. 7. $n_{e \sigma}$ (red and green curves) and $n_{h \sigma}$ (magenta and blue curves) vs $U$ for four different values of $t^{\prime}$, complementary to the values for which the same quantities were shown in Fig. 4 of the main text. In the metallic phases, $n_{e \sigma}>0$ corresponds to electron pockets and $n_{h \sigma}<0$ to hole pockets, whereas in the insulating phases both are zero. Clearly, for $t^{\prime}=0.0 t$ the system is always insulating, whereas $t^{\prime}=0.05 t$ is essentially the first value of $t^{\prime}$ where the PM phase makes an appearance. The panel for $t^{\prime}=0.4 t$ illustrates the shrinking of the range of $U$ corresponding to the AFHM phase with increasing $t^{\prime}$, while the panel for $0.6 t$ illustrates the absence of the BI phase and the direct transition from the PM to the AFI phase for $t^{\prime}>0.5 t$.

phase diagram in Fig. 1 of the main text), while the panel for $0.6 t$ shows the absence of the BI phase and the direct transition from the PM to the AFI phase for $t^{\prime}>0.5 t$.

Finally, Fig. 8 shows the spin-resolved staggered occupancy, $\delta n_{\sigma}=\left(n_{B, \sigma}-n_{A, \sigma}\right) / 2$, and the mean staggered occupancy, $\delta n=1 / 2 \sum_{\sigma} \delta n_{\sigma}$, obtained using DMFT +CTQMC, as a function of $U$ for several values of $t^{\prime}$. These are fully consistent with the nature of the phases and phase transitions shown in Fig. 1 of the main text. An abrupt, first-order change in $\delta n_{\sigma}$ is seen across $U_{c}$ when the magnetic transition is first order. For intermediate values of $t^{\prime}$ where the magnetic transition (PM to FM transition) is continuous or weakly first order, $\delta n_{\sigma}$ also change smoothly across the transition. The slope changes in $\delta n$ vs $U$ at larger $U$ for intermediate values of $t^{\prime}$ are connected with the continuous AFHM to AFI transition.

\section{APPENDIX C: DETAILS OF DMFT + IPT}

In this section, we provide some details regarding the second impurity solver we have used, namely, the iterated perturbation theory (IPT) scheme $[40,43]$, which has the merit of giving semianalytical results directly in the real frequency $\left(\omega^{+} \equiv \omega+i 0^{+}\right)$domain.
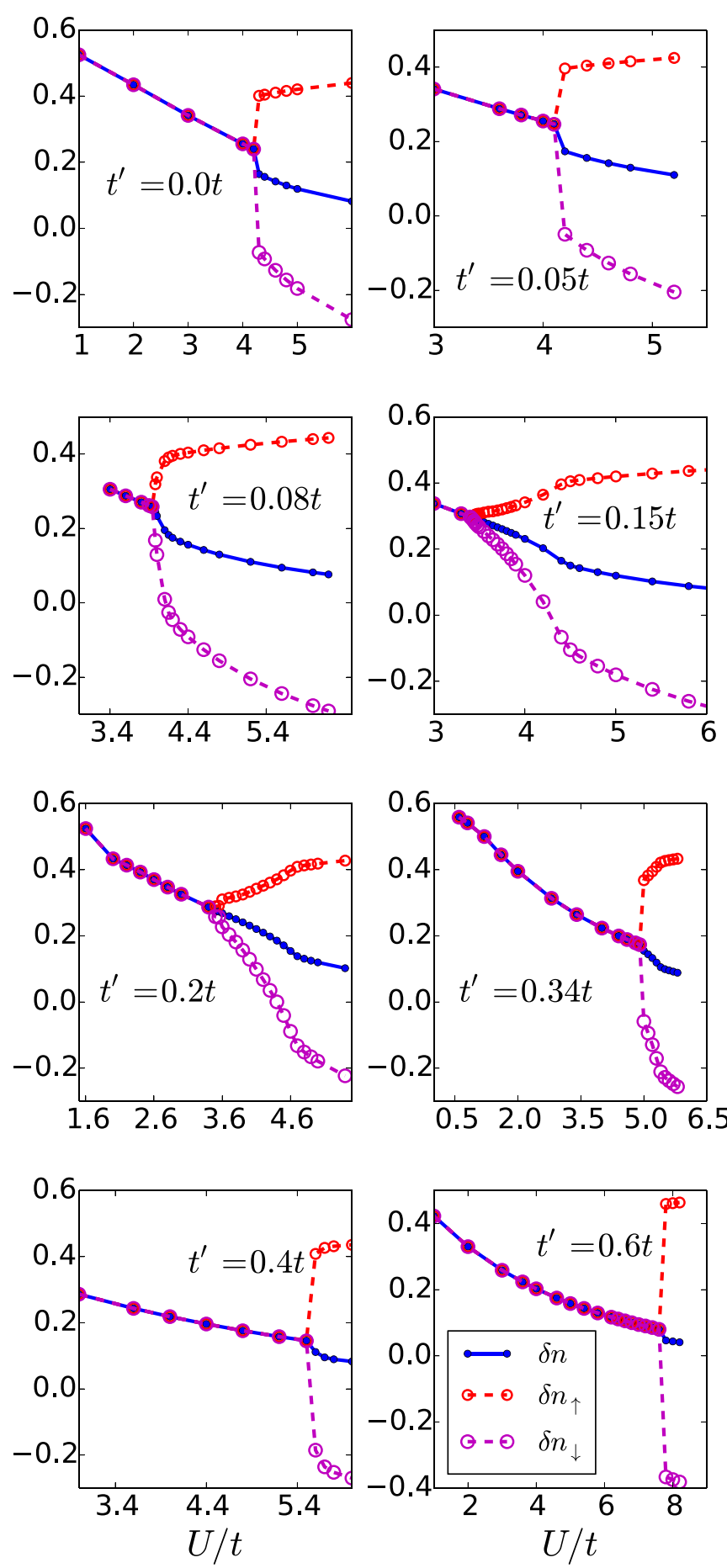

FIG. 8. Spin-resolved staggered occupancy, $\delta n_{\sigma}$, and the mean staggered occupancy, $\delta n$, as functions of $U$ for different values of the second neighbor hopping $\left(t^{\prime}\right)$ obtained using DMFT +CTQMC. These results are for $\left(n_{A}+n_{B}\right) / 2=1$ and $\beta t=50.0, \Delta=1.0 t$. At $t^{\prime}=0.0 t$ and for larger values of $t^{\prime}, \delta n_{\sigma}$ and $\delta n$ have first-order jumps across the magnetic transition. However, at intermediate $t^{\prime}$ values, $\delta n$ seems to change smoothly across the magnetic transition, though it exhibits slope changes at the continuous AFHM-AFI transition.

In the DMFT + IPT method, we start with a guess for the self-energy $\Sigma_{\alpha \sigma}\left(\omega^{+}\right)$, and compute the local Green's 
functions

$$
G_{\alpha \sigma}\left(\omega^{+}\right)=\frac{1}{N} \sum_{\mathbf{k}} G_{\alpha \sigma}^{\sigma}\left(\mathbf{k}, \omega^{+}\right),
$$

where [compare Eqs. (A5) and (A4) above] the momentum, spin, and sublattice resolved Green's functions in the real frequency domain are given by

$$
G_{\alpha \sigma}\left(\mathbf{k}, \omega^{+}\right)=\frac{\zeta_{\bar{\alpha} \sigma}\left(\omega^{+}\right)-\gamma_{\mathbf{k}}^{\prime}}{\left(\zeta_{A \sigma}\left(\omega^{+}\right)-\gamma_{\mathbf{k}}^{\prime}\right)\left(\zeta_{B \sigma}\left(\omega^{+}\right)-\gamma_{\mathbf{k}}^{\prime}\right)-\gamma_{\mathbf{k}}^{2}} .
$$

Here, as before [see discussion after Eq. (A5)], the sum over $\mathbf{k}$ is carried out over a half-point grid in the BZ.

We next determine the "host Green's function" $\mathcal{G}_{\alpha \sigma}$ from the (reversed) Dyson equation

$\mathcal{G}_{\alpha \sigma}^{-1}\left(\omega^{+}\right)=G_{\alpha \sigma}^{-1}\left(\omega^{+}\right)+\Sigma_{\alpha \sigma}\left(\omega^{+}\right) \equiv \omega^{+}-\epsilon_{\alpha}-D_{\alpha \sigma}\left(\omega^{+}\right)$,

where $\epsilon_{A}=\Delta-\mu, \epsilon_{B}=-\Delta-\mu$, and $D_{\alpha \sigma}\left(\omega^{+}\right)$is the analytically continued version of the hybridization function introduced earlier in Eq. (A6).

In the third step, we solve the impurity problem to obtain the self-energy $\Sigma_{\alpha \sigma}\left(\omega^{+}\right)=\Sigma_{\alpha \sigma}\left[\mathcal{G}_{\alpha \sigma}\left(\omega^{+}\right)\right]$. The IPT's use as an impurity solver comes in this step, where one has to obtain the self-energy given the host Green's function.

These three steps are executed iteratively until a selfconsistent solution is obtained. The IPT ansatz is constructed [43] in such a way as to

(a) be exact to second order in $U$ in the weak coupling regime $\left(U / t, U / t^{\prime} \ll 1\right)$;

(b) be exact in the atomic limit $t=t^{\prime}=0$, which also corresponds to the hybridization function being zero;

(c) be exact in the large- $\omega$ limit for all parameter values; and

(d) satisfy the Luttinger-Friedel sum rule.

For this purpose, the self-energy is written [43] in terms of the second order (in $U$ ) self-energy as follows:

$$
\Sigma_{\alpha \sigma}^{\mathrm{IPT}}\left(\omega^{+}\right)=\Sigma_{\alpha \sigma}^{\mathrm{HF}}+\frac{A_{\alpha \sigma} \Sigma_{\alpha \sigma}^{(2)}\left(\omega^{+}\right)}{1-B_{\alpha \sigma} \Sigma_{\alpha \sigma}^{(2)}\left(\omega^{+}\right)} .
$$

Here, as mentioned earlier, $\Sigma_{\alpha \sigma}^{\mathrm{HF}}=U n_{\alpha \bar{\sigma}}$ is the HF selfenergy, and $\Sigma_{\alpha \sigma}^{(2)}$ is the second-order self-energy calculated using a modified host Green's function whose local potential is shifted by an amount $\delta_{\alpha \sigma}$, i.e., $\tilde{\mathcal{G}}_{\alpha \sigma}^{-1}\left(\omega^{+}\right) \equiv \mathcal{G}_{\alpha \sigma}^{-1}\left(\omega^{+}\right)-\delta_{\alpha \sigma}$ :

$$
\begin{aligned}
\Sigma_{\alpha \sigma}^{(2)}\left(\omega^{+}\right)= & U^{2} \prod_{i=1}^{3} \int_{-\infty}^{\infty} d \epsilon_{i}\left[\tilde{\rho}_{\alpha \sigma}\left(\epsilon_{1}\right) \tilde{\rho}_{\alpha \bar{\sigma}}\left(\epsilon_{2}\right) \tilde{\rho}_{\alpha \sigma}\left(\epsilon_{3}\right)\right] \\
& \times \frac{\left[f\left(\epsilon_{1}\right) f\left(-\epsilon_{2}\right) f\left(\epsilon_{3}\right)+f\left(-\epsilon_{1}\right) f\left(\epsilon_{2}\right) f\left(-\epsilon_{3}\right)\right]}{\omega^{+}-\epsilon_{1}+\epsilon_{2}-\epsilon_{3}},
\end{aligned}
$$

where $\tilde{\rho}_{\alpha \sigma}(\omega) \equiv-\operatorname{Im}\left[\tilde{\mathcal{G}}_{\alpha \sigma}\left(\omega^{+}\right)\right] / \pi$ is the spectral function or the density of states (DOS) of the modified host Green's function.

It is straightforward to verify[43] that condition (c) is satisfied if we choose $A_{\alpha \sigma}$ to be

$$
A_{\alpha \sigma}=\frac{n_{\alpha \bar{\sigma}}\left(1-n_{\alpha \bar{\sigma}}\right)}{\tilde{n}_{\alpha \bar{\sigma}}\left(1-\tilde{n}_{\alpha \bar{\sigma}}\right)}
$$

with $\tilde{n}_{\alpha \sigma} \equiv \int_{-\infty}^{0} d \omega \tilde{\rho}_{\alpha \sigma}(\omega)$ is the sublattice and spinresolved occupancy corresponding to the modified host Green's function. The parameter $B_{\alpha \sigma}$, chosen so as to ensure the exact atomic limit of the self-energy, is given by

$$
B_{\alpha \sigma}=\frac{U\left(1-n_{\alpha \bar{\sigma}}\right)-\delta_{\alpha \sigma}}{U^{2} \tilde{n}_{\alpha \bar{\sigma}}\left(1-\tilde{n}_{\alpha \bar{\sigma}}\right)} .
$$

At this stage, the local potential shift $\delta_{\alpha \sigma}$ is still a free parameter, and it can be chosen so as to ensure the Luttinger-Friedel sum rule for the impurity problem, which is equivalent to the following condition [56]:

$$
\int_{-\infty}^{0} \frac{d \omega}{\pi} \operatorname{Im}\left(\frac{\partial \Sigma_{\alpha \sigma}\left(\omega^{+}\right)}{\partial \omega} G_{\alpha \sigma}\left(\omega^{+}\right)\right)=0 .
$$

Figure 5 in Sec. III of the main text, which showed the spinresolved, sublattice-averaged, single-particle density of states (DOS) $\rho_{\sigma}(\omega)$ for $t^{\prime}=0.3 t$ and $\Delta=1.0 t$ for several values of $U / t$, is calculated from the DMFT + IPT converged solutions for the local Green's functions [see Eq. (C1)] as follows:

$$
\rho_{\sigma}(\omega)=\frac{1}{2} \sum_{\alpha} \rho_{\alpha \sigma}(\omega) ; \rho_{\alpha \sigma}(\omega) \equiv-\frac{1}{\pi} \operatorname{Im}\left[G_{\alpha \sigma}\left(\omega^{+}\right)\right] .
$$

\section{APPENDIX D: HARTREE-FOCK MEAN FIELD THEORY OF THE $t-t^{\prime}$ IHM}

When both $U$ and $t$ (and/or $t^{\prime}$ ) are finite, the IHM is no longer exactly solvable. A relatively simple approximate solution can be obtained using the (unrestricted) HartreeFock mean field theory (HF-MFT), where one approximates the Hamiltonian as an effective, self-consistent one-particle Hamiltonian allowing for mean-field order parameters corresponding only to the sublattice and spin resolved occupancies $\left\langle\hat{n}_{\alpha \sigma}\right\rangle \equiv n_{\alpha \sigma}$, or, equivalently (because of the half-filling constraint), to the staggered occupancy $(\delta n)$, the staggered magnetization $\left(m_{s}\right)$ and the uniform magnetization $\left(m_{f}\right)$ defined in Sec. III of the main text. This is achieved by breaking up the interaction term within a mean-field approximation as

$$
U \hat{n}_{i \uparrow} \hat{n}_{i \downarrow} \approx U\left[\left\langle\hat{n}_{i \uparrow}\right\rangle \hat{n}_{i \downarrow}+\hat{n}_{i \uparrow}\left\langle\hat{n}_{i \downarrow}\right\rangle-\left\langle\hat{n}_{i \uparrow}\right\rangle\left\langle\hat{n}_{i \downarrow}\right\rangle\right] .
$$

Furthermore, in this work, we make the simplifying ansatz that $\left\langle\hat{n}_{i \sigma}\right\rangle$ is the same, $n_{A \sigma}$ or $n_{B \sigma}$, for all $\mathrm{A}$ or $\mathrm{B}$ sites respectively. As is shown below in detail, the resulting quadratic Hamiltonian can be diagonalized exactly, leading to four renormalized, spin-dependent, versions of the noninteracting bands, whose dispersions depend on and whose occupancies (plus the half-filling constraint) therefore self-consistently determine the four (spin- and site-resolved) occupancies $n_{\alpha \sigma}$ and the chemical potential $\mu$.

From the numerical solution of these self-consistent equations, we determine the occupancies $n_{\alpha \sigma}$ and hence their linear combinations that correspond to the magnetic order parameters, namely the staggered magnetization $m_{s}$ and the uniform magnetization $m_{f}$, as well as the staggered density $\delta n$, using the relations mentioned in Sec. III of the main text. The HF phases are characterized by the order parameters that are nonvanishing, as well as the nature of the band dispersions. Remarkably, the resulting phase diagram obtained within the HF approximation, depicted in Fig. 9, shows all the same phases as obtained using the 


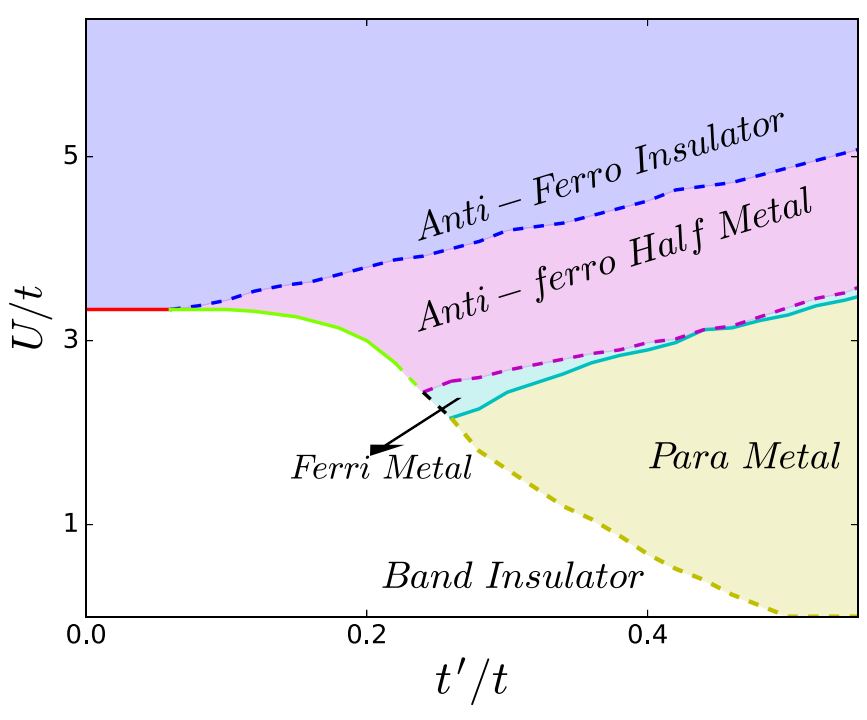

FIG. 9. Phase diagram of the $t-t^{\prime}$ IHM in the $t^{\prime}-U$ plane at halffilling using Hartree-Fock mean-field theory at zero temperature for $\Delta=1.0 t$. For $t^{\prime}<0.06 t$, the system undergoes a direct transition from BI to AFI phase with increase in $U$. For $0.08 t<t^{\prime}<0.24 t$, the system goes from a BI phase to AFI phase via an intermediate AFHM phase upon increase of $U$, whereas for $0.24 t<t^{\prime}<0.26 t$, the system shows three transitions, from BI to FM, from FM to AFHM, and eventually from AFHM to AFI as $U$ is increased. For $0.26 t<t^{\prime}<0.5 t$, the PM phase intervenes between the BI and the other phases, whereas for $t^{\prime}>0.5 t$ there is no BI phase, and the transition is from the PM into other phases. Full lines in the phase diagram indicate first-order transition lines, whereas dashed lines indicate continuous transitions.

much more sophisticated and accurate DMFT +CTQMC calculations, albeit for different ranges of the parameter values. We discuss the details of how we obtain this HF phase diagram and the spectral properties of the various phases in the subsections below.

However, although mean-field versions of all the phases obtained in DMFT +CTQMC calculations show up in the HF calculations as well and the HF-MFT provides useful insights into the nature of the various phases as discussed below, it is a mean-field theory that neglects quantum fluctuations entirely. Hence, as one might expect, it overestimates the regions of stability of the phases with broken symmetry, especially of the AFHM phase, as is clear from a comparison of the two phase diagrams. Needless to say, the inclusion of the quantum fluctuations, especially in the strong correlation regimes, poses considerable challenges, and in this paper we have restricted ourselves to doing this within the DMFT approximation, which correctly includes at least all the local quantum fluctuations, although the challenge of solving the resulting self-consistent impurity problem necessitates the further approximation of CTQMC. The results from these calculations have been discussed in detail in the main text. Needless to say, we expect the DMFT +CTQMC phase diagram to be more accurate, apart from the effects of low-energy, longwavelength spin and particle-hole fluctuations, which are not taken into account in the DMFT (nor are they included in HF-MFT).

\section{HF-MFT calculational details}

Using creation (and annihilation) operators labeled with wave vectors $\mathbf{k}$ according to

$$
\hat{c}_{\alpha \mathbf{k} \sigma}^{\dagger} \equiv \sum_{j \in \alpha} e^{i \mathbf{k} \cdot \mathbf{r}_{j \alpha}} \hat{c}_{j \alpha \sigma}^{\dagger},
$$

where $\alpha=A, B$ is the sublattice index, we can write the (operator part of the) HF Hamiltonian as

$$
\begin{aligned}
\hat{H}_{\mathrm{IHM} ; \mathrm{HF}}= & \sum_{\mathbf{k}, \sigma}\left\{\gamma_{\mathbf{k}}\left(\hat{c}_{A \mathbf{k} \sigma}^{\dagger} \hat{c}_{B \mathbf{k} \sigma}+\text { H.c. }\right)\right. \\
& \left.+A_{\mathbf{k} \sigma} \hat{c}_{A \mathbf{k} \sigma}^{\dagger} \hat{c}_{A \mathbf{k} \sigma}+B_{\mathbf{k} \sigma} \hat{c}_{B \mathbf{k} \sigma}^{\dagger} \hat{c}_{B \mathbf{k} \sigma}\right\} .
\end{aligned}
$$

Here $A_{\mathbf{k} \sigma} \equiv \Delta-\mu+\gamma_{\mathbf{k}}^{\prime}+U n_{A \bar{\sigma}} \quad$ and $\quad B_{\mathbf{k} \sigma} \equiv-\Delta-\mu+$ $\gamma_{\mathbf{k}}^{\prime}+U n_{B \bar{\sigma}}$, with $\gamma_{\mathbf{k}}^{\prime} \equiv-4 t^{\prime} \cos k_{x} \cos k_{y}$, and $\bar{\sigma}$ denotes $\downarrow(\uparrow)$ if $\sigma$ is $\uparrow(\downarrow)$.

Suppose that the one-particle eigenstates of the above Hamiltonian are $\left|d_{\mathbf{k} \sigma}^{ \pm}\right\rangle=\left(P_{A \mathbf{k} \sigma}^{ \pm} \hat{c}_{A \mathbf{k} \sigma}^{\dagger}+P_{B \mathbf{k} \sigma}^{ \pm} \hat{c}_{B \mathbf{k} \sigma}^{\dagger}\right)|0\rangle$ with eigenvalues $\xi_{\mathbf{k} \sigma}^{ \pm}$, which are clearly the effective one-particle band energies of the HF-MFT. Then the eigenvalue equation is

$$
\left(\begin{array}{cc}
A_{\mathbf{k} \sigma} & \gamma_{\mathbf{k}} \\
\gamma_{\mathbf{k}} & B_{\mathbf{k} \sigma}
\end{array}\right)\left(\begin{array}{l}
P_{A \mathbf{k} \sigma}^{ \pm} \\
P_{B \mathbf{k} \sigma}^{ \pm}
\end{array}\right)=\xi_{\mathbf{k} \sigma}^{ \pm}\left(\begin{array}{c}
P_{A \mathbf{k} \sigma}^{ \pm} \\
P_{B \mathbf{k} \sigma}^{ \pm}
\end{array}\right) .
$$

The HF band energies at wave vector $\mathbf{k}$ and for spin $\sigma$ are therefore given by

$$
\begin{aligned}
\xi_{\mathbf{k} \sigma}^{ \pm} & =\frac{1}{2}\left(A_{\mathbf{k} \sigma}+B_{\mathbf{k} \sigma} \pm \sqrt{\left(A_{\mathbf{k} \sigma}-B_{\mathbf{k} \sigma}\right)^{2}+4\left(\gamma_{\mathbf{k}}\right)^{2}}\right), \\
P_{B \mathbf{k} \sigma}^{ \pm 2} & =1 /\left(1+L_{\mathbf{k} \sigma}^{ \pm 2}\right), \\
P_{A \mathbf{k} \sigma}^{ \pm 2} & =L_{\mathbf{k} \sigma}^{ \pm 2} /\left(1+L_{\mathbf{k} \sigma}^{ \pm 2}\right),
\end{aligned}
$$

where $L_{\mathbf{k} \sigma}^{ \pm} \equiv-\gamma_{\mathbf{k}} /\left[A_{\mathbf{k} \sigma}-\xi_{\mathbf{k} \sigma}^{ \pm}\right]$. The particle occupancy at site $\alpha$ for the state $\left(\left|d_{\mathbf{k} \sigma}^{ \pm}\right\rangle\right)$is given by $n_{\alpha \mathbf{k} \sigma}^{ \pm}=\left\langle d_{\mathbf{k} \sigma}^{ \pm}\left|\hat{c}_{\alpha \mathbf{k} \sigma}^{\dagger} \hat{c}_{\alpha \mathbf{k} \sigma}\right| d_{\mathbf{k} \sigma}^{ \pm}\right\rangle=$ $P_{\alpha \mathbf{k} \sigma}^{ \pm 2}$.

The four occupancies at site $\alpha$ and for spin $\sigma$ are determined self-consistently by populating these effective noninteracting bands as per Fermi-Dirac statistics. In the ground state, which is all that we focus on in this paper, only the single-particle states with negative energies are occupied, and hence we get the following four self-consistency relations:

$$
n_{\alpha \sigma}=\sum_{\xi_{\mathbf{k} \sigma}^{ \pm}<0} P_{\alpha \mathbf{k} \sigma}^{ \pm 2} .
$$

For each set of parameter values $\left(U / t, \Delta / t\right.$, and $\left.t^{\prime} / t\right)$, we numerically solve these four equations, along with the halffilling constraint, by iteration, to determine the four (spinand site-resolved) occupancies and the chemical potential $\mu$. To ensure the correctness of our HF calculations, we have benchmarked the results obtained by setting $\Delta=0$ in our calculations against the HF results for the $t-t^{\prime}$ Hubbard model in Ref. [57]. Furthermore, the analytical form of band gap in the noninteracting $t-t^{\prime}$ IHM on a square lattice is $2 \Delta-$ $4 t^{\prime}$. The gap obtained from our numerically calculated density of states (DOS) matches well with this analytical result as $U \rightarrow 0$. 


\section{Staggered and uniform magnetization within HF-MFT}

Especially in the context of understanding the phase diagram in Fig. 9, it is useful to express the HF band dispersion relations in terms of the order parameters $m_{s}, m_{f}$, and $\delta n$. We get

$$
\begin{aligned}
\xi_{\mathbf{k} \sigma}^{ \pm} \equiv & \tilde{\epsilon}_{\mathbf{k} \sigma}^{ \pm}-\mu=-4 t^{\prime} \cos k_{x} \cos k_{y}+\frac{U}{2}-\frac{\sigma U m_{f}}{2}-\mu \\
& \pm \sqrt{\left[\Delta-U\left(\frac{\delta n+\sigma m_{s}}{2}\right)\right]^{2}+\left[\gamma_{\mathbf{k}}\right]^{2}}
\end{aligned}
$$

By comparing with the noninteracting band dispersion in En. (2) given in Sec. II of the main text, clearly one can interpret these results in terms of an effective spin-dependent staggered potential $\tilde{\Delta}_{\sigma}=\Delta-U\left(\delta n+\sigma m_{s}\right) / 2$ and an effective spin-dependent uniform potential $-\sigma U m_{f} / 2$. The effective band gap $\left(\tilde{E}_{\mathrm{gap}, \sigma}\right)$ for electrons with spin $\sigma$, determined by the difference between the bottom of the conduction band $\left(\xi_{\mathbf{K}^{\prime} \sigma}^{+}\right)$and the top of the valence band $\left(\xi_{\mathbf{K} \sigma}^{-}\right)$, is now $\tilde{E}_{\text {gap }, \sigma}=2\left|\tilde{\Delta}_{\sigma}\right|-4 t^{\prime}$, which is interaction and spin dependent.

First, consider the HF phase diagram (Fig. 9) in the paramagnetic regime, where both the staggered magnetization, $m_{s}$, and the uniform magnetization, $m_{f}$, are zero. Hence, $\tilde{\Delta}_{\uparrow}=$ $\tilde{\Delta}_{\downarrow} \equiv \tilde{\Delta}=\Delta-U(\delta n / 2)$. Consequently the single-particle excitation band gap $\tilde{E}_{\text {gap }, \sigma}$ is also spin symmetric, being given by $\left(2|\tilde{\Delta}|-4 t^{\prime}\right)$. Over the parameter range where $U$ and $t^{\prime}$ are sufficiently small that $\tilde{E}_{\text {gap }}$ remains positive, we get an insulating phase which is adiabatically connected to the noninteracting BI phase, and hence we use the same label for it.

As $U$ increases, to leading order in $U$ the insulating gap decreases linearly with $U$ because of the density difference between the two sublattices, $\delta n$ induced by the ionic potential $\Delta$; however, this decrease gets suppressed at higher orders in $U$ even within $\mathrm{HF}$ theory because $U$ promotes single occupancy of lattice sites, leading to a reduction in $\delta n$. If the first effect were to remain dominant, one would expect that as one increases $U$ further, $\tilde{E}_{\text {gap }}$ might go to zero at a certain $U$, whence one would get a band insulator to metal transition. In contrast, when $t^{\prime}=0$, one finds that the system never goes to a paramagnetic metallic state within the HF theory, i.e., $\tilde{E}_{\text {gap }}$ decreases monotonically but remains nonzero for all $U$. But, as mentioned in Sec. I of the main text, more accurate calculations using DMFT, which include local correlation effects beyond $\mathrm{HF}$, do lead to a metallic state, as pointed out first by Garg et al. [19]. Later works [23-26,36] showed that this metallic solution is pre-empted, however, by AF ordering [26], leading to a direct transition from BI to the AFI state.

As is clear from Fig. 9, this inability of the paramagnetic metal (PM) phase to become viable before magnetic order sets in continues to hold within the HF-MFT even when $t^{\prime} \neq 0$, as long as $t^{\prime}<0.26 t$ for $\Delta=1.0 t$. As discussed in detail in the main text, one of the main results of the work presented in this paper is that when $t^{\prime}$ is sufficiently large $\tilde{E}_{\text {gap }}$ does go to zero within the paramagnetic sector, leading to a robust paramagnetic BI to PM transition and a stable correlationinduced PM phase, both within the HF-MFT and the more accurate DMFT +CTQMC. While the threshold $t^{\prime}$ for the viability of the PM phase is close to $0.26 t$ for, $\Delta=1.0 t$ within
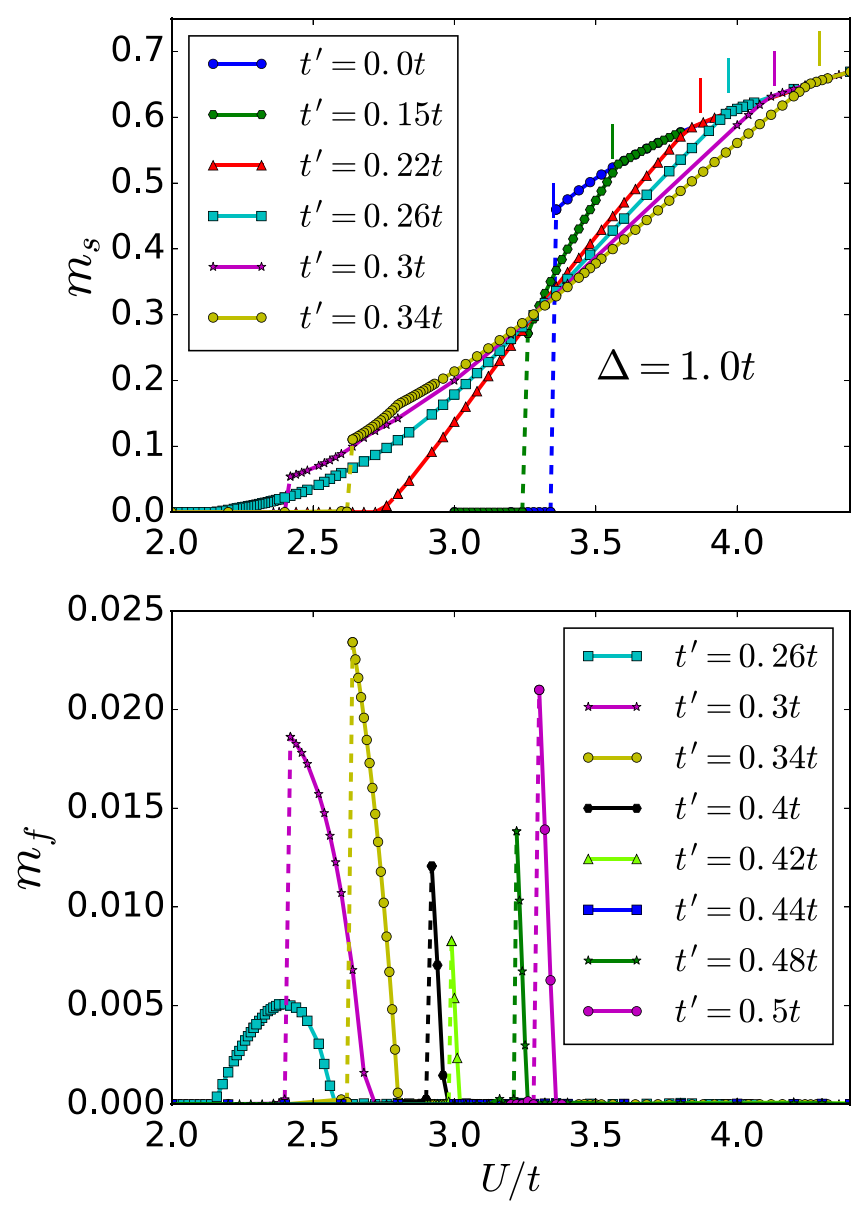

FIG. 10. HF theory results for the staggered magnetization $m_{s}$ (a) and the uniform magnetization $m_{f}$ (b) as a function of the Hubbard interaction $U$ for different values of the second neighbor hopping $\left(t^{\prime}\right)$ for $\left(n_{A}+n_{B}\right) / 2=1, T=0$, and $\Delta=1.0 t$. The magnetic transition is first order except for $t^{\prime}$ in the range from $0.215 t$ to $0.26 t$, where it is a continuous transition. Note that the values of $m_{f}$ are rather small and exist only for relatively larger values of $t^{\prime}$ (for $t^{\prime} \geqslant 0.24 t$ ) and only for limited ranges of $U$, corresponding to the magenta shaded region in Fig. 9. The FM-AFHM and the AFHMAFI transitions are continuous and show up as kinks (changes of slope) in the $m_{s}$ vs $U / t$ curves.

the HF-MFT, it is much smaller, a bit less than $0.05 t$, within DMFT +CTQMC, consistent with the results for $t^{\prime}=0$.

Next, we discuss results from the unrestricted HF-MFT when the spin symmetry breaking is allowed. Figures 10(a) and 10(b) show the magnetic order parameters $m_{s}$ and $m_{f}$ at zero temperature within the HF-MFT as functions of $U$ for different values of $t^{\prime}$. As $U$ increases, the staggered magnetization $m_{s}$ turns on at a threshold value $U_{c}\left(t^{\prime}\right)$, which depends on $t^{\prime}$. At the smaller $t^{\prime}$ values $\left(t^{\prime}<0.215 t\right.$ for $\left.\Delta=1.0 t\right)$, the magnetic transition is a first-order AF transition, with $m_{f}=0$ for all values of $U$. As $t^{\prime}$ increases, the discontinuity in $m_{s}$ across the transition decreases. We find that the transition is continuous for $0.215 t<t^{\prime}<0.265 t$ and then again becomes first order as $t^{\prime}$ increases beyond $0.26 t$, indicating the presence of multicritical points at $\left(U=2.82 t, t^{\prime}=0.215 t\right)$ and $\left(U=2.16 t, t^{\prime}=0.26 t\right)$. The net magnetization $m_{f}$ seems to turn on for the first time for $t^{\prime} \approx 0.24 t$ when the magnetic 


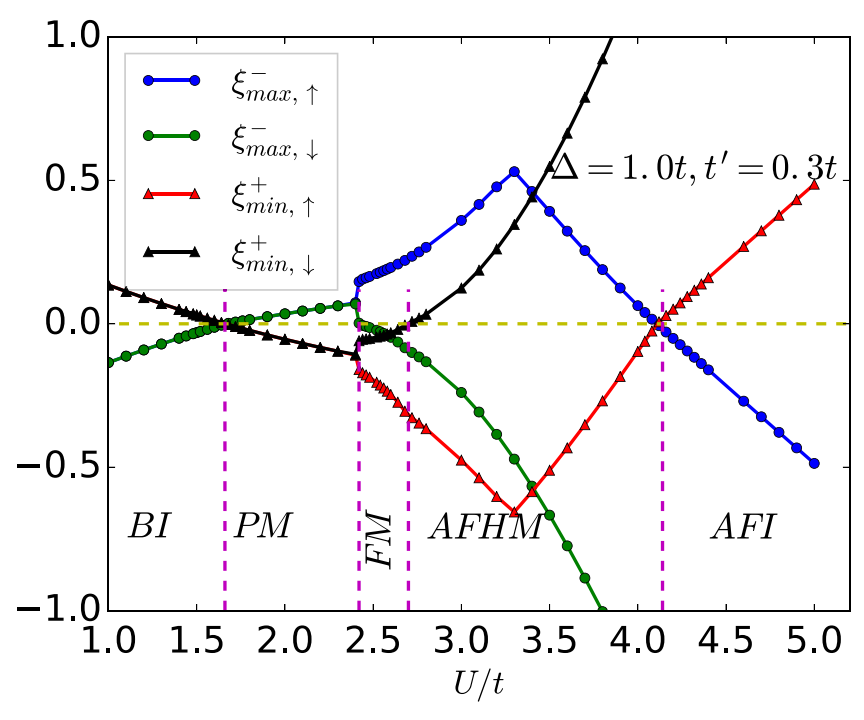

FIG. 11. Maxima of the valence (-) and minima of the conduction $(+) \mathrm{HF}$ bands as measured from the chemical potential $(\mu)$ for both the spins plotted as functions of $U / t$ for $t^{\prime}=0.3 t$.

transition is continuous. For $t^{\prime}>0.26 t, m_{f}$ also shows a firstorder jump, indicating that the magnetic transition has become first order in nature. The first-order nature of the transition in $m_{s}$ as a function of $U$ in Fig. 10 is consistent with earlier results for $t^{\prime}=0$ on the square lattice [36] and on the Bethe lattice [26], and differ from the results in Ref. [23]. Similar changes in the order of magnetic transition with increase in $t^{\prime}$ have been observed [57] in the $t-t^{\prime}$ Hubbard model. [Note, however, that the FM-AFHM and the AFHM-AFI transitions are continuous (see below) and can be identified by kinks (changes of slope) in the $m_{s}$ versus $U / t$ curves. Clearly, these lead to other multicritical points in the phase diagram.]
We note that just as in the DMFT + CTQMC phase diagram, and for the same reasons as discussed earlier, $U_{c}$ within the HF-MFT also has a nonmonotonic dependence on $t^{\prime}$, initially decreasing with increasing $t^{\prime}$, reaching a minimum (around $t^{\prime}=0.26 t$ in HF-MFT), and then increasing again. The initial decrease in $U_{c}$ correlates with the reduction in the effective band gap, $\tilde{E}_{\text {gap }}$ as $t^{\prime}$ increases, consistent $[26,58]$ with the dependence of $U_{c}$ for the BI-AFI transition on $\Delta$ for $t^{\prime}=0$. Another aspect of the second neighbor hopping is that $t^{\prime}$ introduces frustration against AFM ordering [57,59], which should effectively increase the $U_{c}$ required for ordering. These two competing effects of $t^{\prime}$ are responsible for the observed nonmonotonic trend of $U_{c}$ with increasing $t^{\prime}$ both within the HF and the DMFT methods.

\section{Band structure within HF-MFT}

Additional and interesting aspects of the magnetically ordered phases emerge from an examination of the selfconsistent HF band dispersions and their gaps and overlaps. Figure 11 shows the minima of the upper (conduction) band(s), $\left(\xi_{\min , \sigma}^{+}\right)$, and the maxima of the lower (valence) band(s), $\left(\xi_{\max , \sigma}^{-}\right)$, measured relative to the chemical potential $\mu$ and for each spin channel, as functions of $U$ for $t^{\prime}=0.3 t$, whereas Fig. 12 shows the band dispersions along specific paths (mentioned in the caption) in the Brillouin zone for five representative values of $U$ chosen to correspond to the five different phases seen in Fig. 9 for $t^{\prime}=0.3 t$ as $U$ is increased.

In the BI and PM phases, both of which are paramagnetic, with $m_{s}=0=m_{f}$, the band dispersions and the maxima and minima are spin symmetric. In the BI phase, obtained for $U / t<1.7, \xi_{\min }^{+}>0$ and $\xi_{\max }^{-}<0$, corresponding to an empty conducting band and a filled valence band with a gap between them. This is also clearly seen in Fig. 12(a). As $U$ increases, $\xi_{\min }^{+}$decreases as $|\tilde{\Delta}|-\tilde{\mu}$, whereas $\xi_{\max }^{-}$increases as $4 t^{\prime}-|\tilde{\Delta}|-\tilde{\mu}$, where $\tilde{\mu} \equiv \mu-U / 2$ is zero when $t^{\prime}$ is

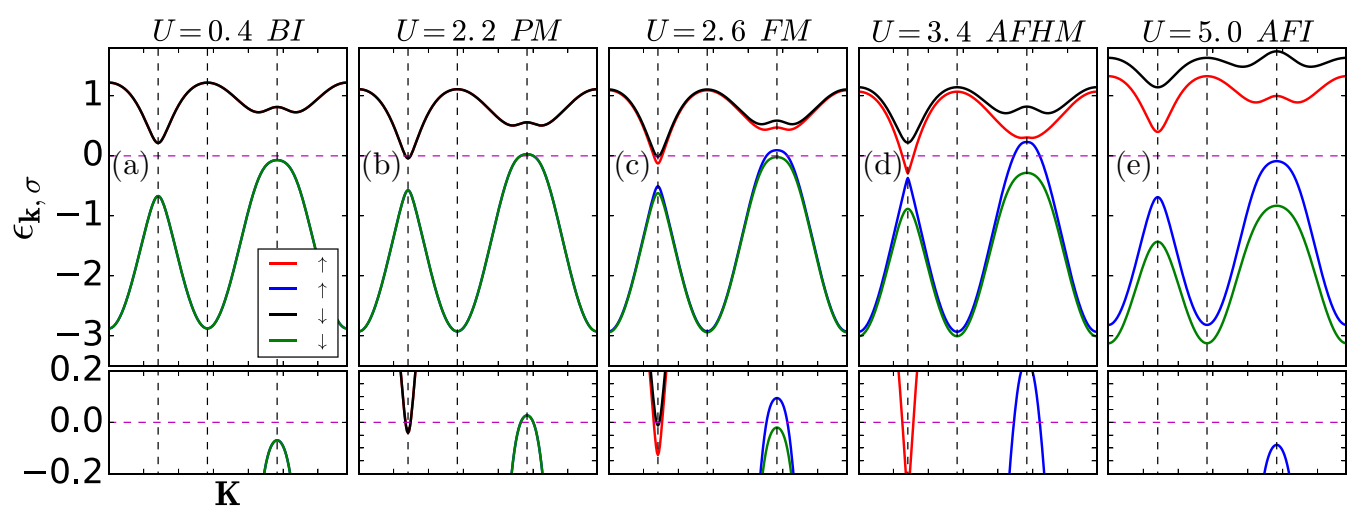

FIG. 12. Effective band dispersions, calculated using HF-MFT, plotted along the path in the Brillouin zone corresponding to $(0,0) \rightarrow$ $(\pi / 2, \pi / 2) \rightarrow(\pi, \pi) \rightarrow(0, \pi) \rightarrow(0,0)$ (vertical doted lines indicate the break points) for different values of $U$ at $\Delta=1.0 t$ and $t^{\prime}=0.3 t$ Enlarged plots of the dispersions near zero frequency are presented in the lower panels. (a) For $U=0.4 t$, the upper band has a minimum at $(\pi / 2, \pi / 2)$ and the lower band has a maximum at $(0, \pi)$. The chemical potential lies within the gap. Hence, the system is in the (paramagnetic) BI phase. (b) For $U=2.2 t$, both the upper and the lower bands cross the chemical potential. The upper band has an electron pocket at $(\pi / 2, \pi / 2)$ while the lower band has a hole pocket at $(0, \pi)$. This is the paramagnetic metallic phase. (c) For $U=2.6 t$, the band structure is different for $\uparrow$ and $\downarrow$ spins. The upper bands for both the spins cross the chemical potential, whereas only the lower $\downarrow$ spin band crosses the chemical potential. Hence, we get a ferrimagnetic metallic state. (d) For $U=3.4 t$, the $\uparrow$ spin bands cross the chemical potential whereas the $\downarrow$ bands are gapped out, corresponding to the AFHM state. (e) For $U=5.0 t$, bands for both the spins are gapped out, and the system is in an AFI phase. 
zero. The BI-PM transition occurs when the two lines cross 0 (simultaneously because of the half-filling constraint), at $U / t=1.7$, as is clear from Fig. 11. In the PM phase (obtained for $1.7<U / t<2.4$ ), the two switch signs, with $\xi_{\min }^{+}<0$ and $\xi_{\max }^{-}>0$, corresponding to band overlap, electron pockets near the conduction band minima, and hole pockets near the valence band maxima, as can be clearly seen in Fig. 12(b). (See Fig. 4 for the corresponding scenario obtained using the MDF within DMFT +CTQMC.)

As $U$ increases past $U_{c}\left(U_{c}=2.4 t\right.$ for $\left.t^{\prime}=0.3 t\right)$, the spin symmetry is broken and antiferromagnetic order sets in. The effective staggered potential $\tilde{\Delta}_{\sigma}$ is spin dependent, leading to spin split bands, maxima, and minima. For a positive $m_{s}$, corresponding to the A site being preferentially occupied by up spins $\left(\tilde{\Delta}_{\uparrow}<\tilde{\Delta}_{\downarrow}\right)$, we find that there is a small range of $U$ past $U_{c}$ where the down-spin bands have only electron pockets $\left(\xi_{\min , \downarrow}^{+}<0\right.$ but $\left.\xi_{\text {max }, \downarrow}^{-}<0\right)$, while the up-spin bands have both electron and hole pockets $\left(\xi_{\min , \uparrow}^{+}<0\right.$ and $\left.\xi_{\max , \uparrow}^{-}>0\right)$, as is also clear Fig. 12(c). Hence, $m_{f} \neq 0$, which corresponds to the FM phase, although the $m_{f}$ values are rather small [Fig. 10(b)]. The first-order nature of the PM-FM transition is also evident in Fig. 11. Further increase of $U$ leads to $\xi_{\min , \downarrow}^{+}$ increasing and changing sign, transiting to a broad range of $U$ where the $\uparrow$ spin bands overlap increasingly, while the $\downarrow$ spin bands are fully gapped, corresponding to the AFHM phase, with a band structure as in Fig. 12(d). The overlap of the $\uparrow$ spin bands is a nonmonotonic function of $U$, however, starts decreasing past a threshold $U$, and crosses zero again, resulting in a transition from the AFHM phase to the AFI phase, where the $\uparrow$ spin bands are gapped as well, but with a smaller gap compared to the $\downarrow$-spin bands, as is clear from Fig. 11, and also from the band structure in Fig. 12(e). The entire sequence of phases and phase transitions is in accordance with the HF-MFT phase diagram in Fig. 9.

\section{Single-particle density of states within HF-MFT}

All of the features of the phases and phase transitions discussed above can be seen more directly in the single-particle density of states (DOS) for real frequencies, which can be calculated exactly within the HF approximation. Specifically, Fig. 13 shows the results of our calculations for the spin-resolved single-particle DOS averaged over the two sublattices, $\rho_{\sigma}(\omega)$, calculated using the same equation [Eq. (C9) of Appendix C] as used in the case of the DMFT + IPT solution discussed earlier, except that now $G_{\alpha \sigma}(\omega)$ is the HF-MFT Green's function on sublattice $\alpha$.

The top panels in Fig. 13 show the DOS for $t^{\prime}=0.3 t$ and $\Delta=1.0 t$ for four of the values of $U$ in Fig. 12. For $U=2.2 t$, in the PM phase, where the system has spin symmetry (with $\left.m_{s}=m_{f}=0\right)$, the DOS for the two spin channels is the same and finite at the Fermi level (corresponding to $\omega=0$ ). For $U=2.6 t$, in the FM phase, where both the magnetic order parameters are nonzero, the DOS is spin asymmetric but finite at the Fermi level for both the spin channels. For $U=3.4 t, A_{\downarrow}(\omega)$ has a gap around the Fermi level, while $A_{\uparrow}(\omega=0)$ is finite. Because of the half-filling constraint, this necessarily implies that $m_{f}=0$, so this is the AFHM phase. For $U=5.0 t$, gaps in the DOS are present in both the spin channels, corresponding to the AF (Mott) insulating (AFI)
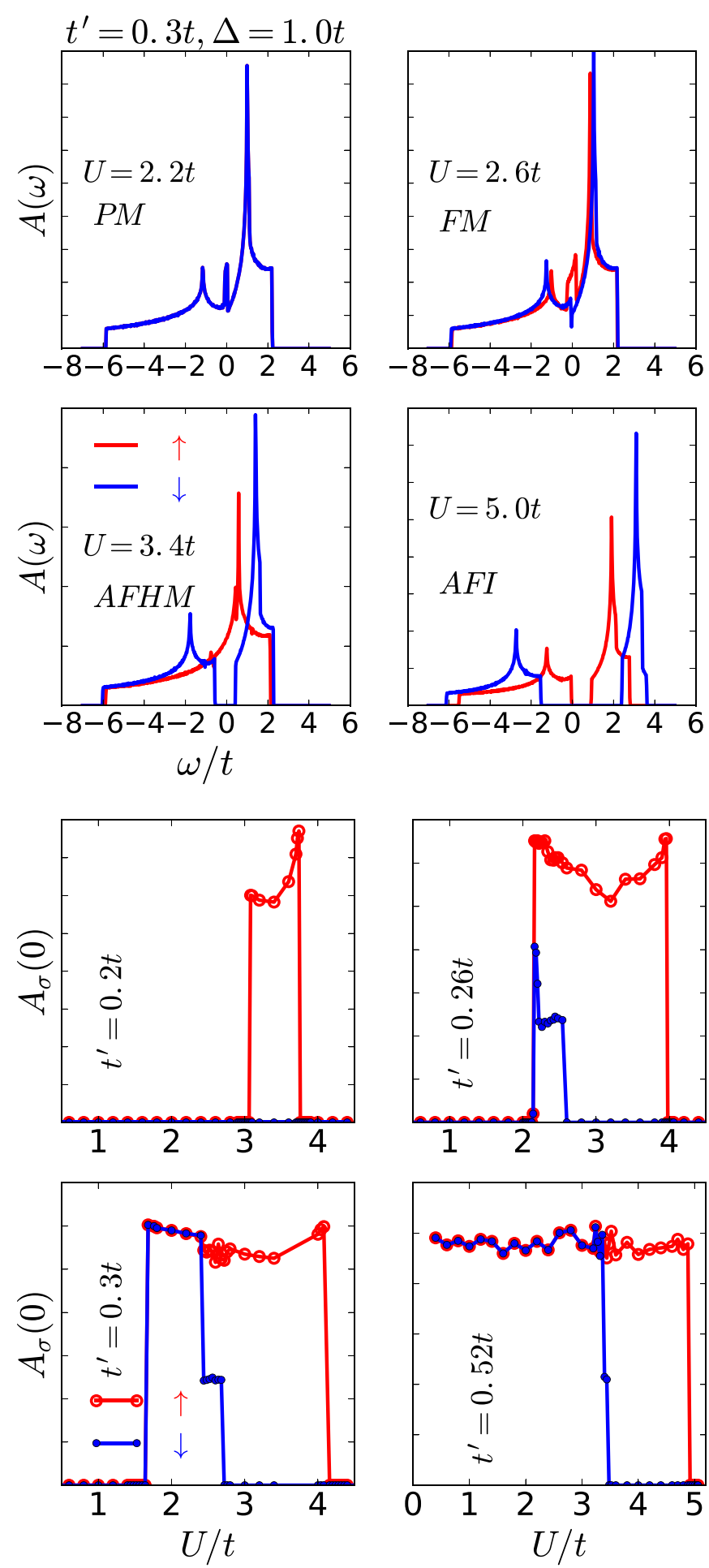

FIG. 13. The top four panels show the HF spectral function $A_{\sigma}(\omega)$ vs $\omega$ for $t^{\prime}=0.3 t$ for four $U$ values. The bottom set of four panels present $A_{\sigma}(\omega=0)$ vs $U$ for four $t^{\prime}$ values. As $U$ increases for $t^{\prime}=0.3 t$, one can see that the system undergoes a transition from a BI to a paramagnetic metal, followed by transitions into ferrimagnetic metallic and AF half-metallic phases, and eventually becomes an AFI.

phase. The lower panels of Fig. 13 show the evolution of the DOS at the Fermi level, $A_{\sigma}(\omega=0)$, as a function of $U$ for four illustrative values of $t^{\prime}$, bringing out the sequence of phases 
and phase transitions that occur as $U$ increases consistent with the complete phase diagram in Fig. 9. The existence (for different ranges of $U$ ) of the $\mathrm{BI}$ (for smaller $U$ values) as well as AFI (for larger $U$ values) phases where the DOS in both spin channels is zero, and the AF half-metal where
$A_{\downarrow}(\omega=0)=0$ but $A_{\uparrow}(\omega=0) \neq 0$ is clearly evident. For $t^{\prime}=$ $0.3 t$, the PM phase, where $A_{\uparrow}(\omega=0)=A_{\downarrow}(\omega=0) \neq 0$, and the FM phase, where $A_{\uparrow}(\omega=0) \neq A_{\downarrow}(\omega=0) \neq 0$, appear as well. For $t^{\prime}=0.52 t$, the PM phase is present even at $U=0$, and there is no BI phase.
[1] M. Imada, A. Fujimori, and Y. Tokura, Rev. Mod. Phys. 70, 1039 (1998); P. A. Lee, N. Nagaosa, and X. G. Wen, ibid. 78, 17 (2006).

[2] X. Hu, Adv. Mater. 24, 294 (2012); X. Li and J. Yang, Natl. Sci. Rev. 3, 365 (2016).

[3] W. E. Pickett and J. S. Moodera, Phys. Today 54(5), 39 (2001); Edited by I. Galanakis and P. H. Dederich, Half-Metallic Alloys: Fundamentals and Applications, Lecture Notes in Physics Vol. 676 (Springer, New York, 2005); M. I. Katsnelson, V. Y. Irkhin, L. Chioncel, A. I. Lichtenstein, and R. A. de Groot, Rev. Mod. Phys. 80, 315 (2008); Edited by C. Felser and G. Fetcher, Spintronics: From Materials to Devices (Springer, Dordrecht, 2013); A. Hirohata, K. Yamada, Y. Nakatani, I. Prejbeanu, B. Diény, P. Pirro, and B. Hillebrands, J. Magn. Magn. Mater. 509, 166711 (2020).

[4] R. A. de Groot, A. M. van der Kraan, and K. H. J. Buschow, J. Magn. Magn. Mater. 61, 330 (1986); R. A. de Groot, Phys. B (Amsterdam, Neth.) 172, 45 (1991); H. van Leuken and R. A. de Groot, Phys. Rev. Lett. 74, 1171 (1995); W. E. Pickett, Phys. Rev. B 57, 10613 (1998); J. H. Park, S. K. Kwon, and B. I. Min, ibid. 65, 174401 (2002); W. E. Pickett and H. Eschrig, J. Phys.: Condens. Matter 19, 315203 (2007); I. Galankis, K. Ozdogan, E. Sasioglu, and B. Aktas, Phys. Status Solidi A 205, 1036 (2008).

[5] D. Kodderitzsch, W. Hergert, Z. Szotek, and W. M. Temmerman, Phys. Rev. B 68, 125114 (2003); W. B. Zhang, N. Yu, W. Y. Yu, and B. Y. Tang, Eur. Phys. J. B 64, 153 (2008).

[6] Y. M. Nie and X. Hu, Phys. Rev. Lett. 100, 117203 (2008).

[7] K.-I. Kobayashi, T. Kimura, H. Sawada, K. Terakura, and Y. Tokura, Nature (London) 395, 677 (1998); D. D. Sarma, P. Mahadevan, T. Saha-Dasgupta, S. Ray, and A. Kumar, Phys. Rev. Lett. 85, 2549 (2000); O. Erten, O. N. Meetei, A. Mukherjee, M. Randeria, N. Trivedi, and P. Woodward, ibid. 107, 257201 (2011); S. Li, P. Chen, and B. Liu, AIP Adv. 3, 012107 (2013).

[8] The model was first proposed for describing the neutral-ionic transition in charge-transfer organic chains [9-12]. Later, it was invoked for understanding polarization phenomena in perovskite materials [13-17] and covalent insulators such as FeSi and $\mathrm{FeSb}_{2}[18]$.

[9] J. Hubbard and J. B. Torrance, Phys. Rev. Lett. 47, 1750 (1981).

[10] J. B. Torrance, A. Girlando, J. J. Mayerle, J. I. Crowley, V. Y. Lee, P. Batail, and S. J. LaPlaca, Phys. Rev. Lett. 47, 1747 (1981).

[11] J. B. Torrance, J. E. Vazquez, J. J. Mayerle, and V. Y. Lee, Phys. Rev. Lett. 46, 253 (1981).

[12] M. H. Lemée-Cailleau, M. Le Cointe, H. Cailleau, T. Luty, F. Moussa, J. Roos, D. Brinkmann, B. Toudic, C. Ayache, and N. Karl, Phys. Rev. Lett. 79, 1690 (1997).

[13] T. Egami, S. Ishihara, and M. Tachiki, Science 261, 1307 (1993).
[14] S. Ishihara, T. Egami, and M. Tachiki, Phys. Rev. B 49, 8944 (1994).

[15] R. Resta and S. Sorella, Phys. Rev. Lett. 74, 4738 (1995).

[16] M. E. Torio, A. A. Aligia, and H. A. Ceccatto, Phys. Rev. B 64, 121105(R) (2001).

[17] G. Ortiz, P. Ordejón, R. M. Martin, and G. Chiappe, Phys. Rev. B 54, 13515 (1996).

[18] J. Kuneš and V. I. Anisimov, Phys. Rev. B 78, 033109 (2008).

[19] A. Garg, H. R. Krishnamurthy, and M. Randeria, Phys. Rev. Lett. 97, 046403 (2006).

[20] N. Paris, K. Bouadim, F. Hebert, G. G. Batrouni, and R. T. Scalettar, Phys. Rev. Lett. 98, 046403 (2007).

[21] A. T. Hoang, J. Phys.: Condens. Matter 22, 095602 (2010).

[22] L. Craco, P. Lombardo, R. Hayn, G. I. Japaridze, and E. MullerHartmann, Phys. Rev. B 78, 075121 (2008).

[23] K. Byczuk, M. Sekania, W. Hofstetter, and A. P. Kampf, Phys. Rev. B 79, 121103(R) (2009).

[24] H.-M. Chen, H. Zhao, H.-Q. Lin, and C.-Q. Wu, New J. Phys. 12, 093021 (2010).

[25] A. Garg, H. R. Krishnamurthy, and M. Randeria, Phys. Rev. Lett. 112, 106406 (2014).

[26] S. Bag, A. Garg, and H. R. Krishnamurthy, Phys. Rev. B 91, 235108 (2015).

[27] Extensive studies of the IHM in one dimension [28-35], where the AF order is suppressed due to quantum fluctuations, suggest a bond-ordered (BO) insulating phase (and not a PM phase) intervening between the BI and MI phases. In two and higher dimensions, the existence and nature of the intermediate phase is still controversial despite several attempts based on various approximate methods of solving the $t$-IHM [20,21,24,36,37].

[28] C. D. Batista and A. A. Aligia, Phys. Rev. Lett. 92, 246405 (2004).

[29] M. Fabrizio, A. O. Gogolin, and A. A. Nersesyan, Phys. Rev. Lett. 83, 2014 (1999).

[30] A. P. Kampf, M. Sekania, G. I. Japaridze, and P. Brune, J. Phys.: Condens. Matter 15, 5895 (2003).

[31] S. R. Manmana, V. Meden, R. M. Noack, and K. Schönhammer, Phys. Rev. B 70, 155115 (2004).

[32] H. Otsuka and M. Nakamura, Phys. Rev. B 71, 155105 (2005).

[33] Y. Z. Zhang, C. Q. Wu, and H. Q. Lin, Phys. Rev. B 67, 205109 (2003).

[34] M. E. Torio, A. A. Aligia, G. I. Japaridze, and B. Normand, Phys. Rev. B 73, 115109 (2006).

[35] G. I. Japaridze, R. Hayn, P. Lombardo, and E. MüllerHartmann, Phys. Rev. B 75, 245122 (2007).

[36] S. S. Kancharla and E. Dagotto, Phys. Rev. Lett. 98, 016402 (2007).

[37] H.-F. Lin, H.-D. Liu, H.-S. Tao, and W.-M. Liu, Sci. Rep. 5, 9810 (2015).

[38] However, doping away from half filling leads to a ferrimagnetic half-metallic phase [25]. 
[39] As is clear from the phase diagram in Fig. 1 and discussed in detail in Sec. III, on either side of the range of $t^{\prime}$ mentioned above, there is no FM phase, and the transition sequence is BI $\rightarrow \mathrm{PM} \rightarrow \mathrm{AFHM} \rightarrow \mathrm{AFI}$. For $t^{\prime}$ small or close to $\Delta / 2$, there is no AFHM phase, and the transition sequence is $\mathrm{BI} \rightarrow$ AFI and $\mathrm{BI} \rightarrow \mathrm{PM} \rightarrow$ AFI respectively.

[40] A. Georges, G. Kotliar, W. Krauth, and M. J. Rozenberg, Rev. Mod. Phys. 68, 13 (1996).

[41] T. Prushke, M. Jarrell, and J. K. Freericks, Adv. Phys. 44, 187 (1995).

[42] E. Gull, A. J. Millis, A. I. Lichtenstein, A. N. Rubtsov, M. Troyer, and P. Werner, Rev. Mod. Phys. 83, 349 (2011); P. Werner, A. Comanac, L. de'Medici, M. Troyer, and A. J. Millis, Phys. Rev. Lett. 97, 076405 (2006).

[43] H. Kajueter and G. Kotliar, Phys. Rev. Lett. 77, 131 (1996).

[44] DMFT+IPT has led to qualitatively the same results as obtained using other DMFT impurity solvers, as well as even other techniques. One of the known limitations of IPT is that it tends to overestimate the effective Kondo temperature for the self-consistent Anderson impurity problem, but in the broken symmetry phases with antiferromagnetic or ferrimagnetic order which we are focusing on in our paper, there is a (Zeeman) molecular field in the impurity problems and the Kondo effect is suppressed. For the half-filled IHM for $t^{\prime}=0$, a detailed comparison of CTQMC and IPT solvers is discussed in Ref. [26]. Results obtained within both the methods have good qualitative and quantitative consistency in the intermediate to strong coupling regime at zero temperature as well as at finite temperatures.

[45] It is to be noted that, although our calculations are done on the 2-d square lattice, given that they are done within the framework of DMFT which works best in the limit of large dimensions, our results are really representative of three- or higher dimensional systems with bounded band structures. In an actual 2-d system, at any finite temperature, long wavelength fluctuations of the magnetic order parameters, which are not included in the DMFT, will destabilize the magnetic phases we have reported.

[46] Perhaps the best known way of doing the analytic continuation is to use the maximum entropy method (MEM) [e.g., see M. Jarrell and J. E. Gubernatis, Phys. Rep. 269, 133 (1996); R. K.
Bryan, Eur. Biophys. J. 18, 165 (1990)]. However, the method requires an initial guess for the DOS, which can, in principle, incorrectly bias the real (low) frequency data required to sharply distinguish between a metal and an insulator.

[47] M. Greiner, C. A. Regal, and D. S. Jin, Nature (London) 426, 537 (2003); M. Griener, I. Bloch, O. Mandel, T. W. Hänsch, and T. Esslinger, Phys. Rev. Lett. 87, 160405 (2001); M. Kohl, H. Moritz, T. Stöferle, K. Günter, and T. Esslinger, ibid. 94, 080403 (2005); C. A. Regal, M. Greiner, S. Giorgini, M. Holland, and D. S. Jin, ibid. 95, 250404 (2005).

[48] J. C. Campuzano, M. R. Norman, M. Randeria, in Physics of Superconductors, edited by K. H. Bennemann and J. B. Ketterson (Springer, Berlin, 2004), pp. 167-273.

[49] R. Nandkishore, G. W. Chern, and A. V. Chubukov, Phys. Rev. Lett. 108, 227204 (2012); J. Yuan, D. H. Xu, H. Wang, Y. Zhou, J. H. Gao, and F. C. Zhang, Phys. Rev. B 88, 201109(R) (2013).

[50] T. A. Maier, M. Jarrell, T. Pruschke, and M. H. Hettler, Rev. Mod. Phys. 77, 1027 (2005); G. Rohringer, H. Hafermann, A. Toschi, A. A. Katanin, A. E. Antipov, M. I. Katsnelson, A. I. Lichtenstein, A. N. Rubtsov, and K. Held, ibid. 90, 025003 (2018).

[51] We note that in the $t-t^{\prime}$ Hubbard model there are also interesting instabilities leading to the formation of incommensurate spiral spin density waves (e.g., see Ref. [52]). These are worth investigating in the $t-t^{\prime}$ IHM as well.

[52] H. R. Krishnamurthy, C. Jayaprakash, S. Sarker, and W. Wenzel, Phys. Rev. Lett. 64, 950 (1990).

[53] M. Messer, R. Desbuquois, T. Uehlinger, G. Jotzu, S. Huber, D. Greif, and T. Esslinger, Phys. Rev. Lett. 115, 115303 (2015).

[54] E. V. Castro, K. S. Novoselov, S. V. Morozov, N. M. R. Peres, J. M. B. Lopes dos Santos, J. Nilsson, F. Guinea, A. K. Geim, and A. H. Castro Neto, Phys. Rev. Lett. 99, 216802 (2007).

[55] K. Haule, Phys. Rev. B 75, 155113 (2007).

[56] A. C. Hewson, The Kondo Problem to Heavy Fermions (Cambridge University Press, Cambridge, UK, 1997).

[57] W. Hofstetter and D. Vollhardt, Ann. Phys. 7, 49 (1998).

[58] A. Euverte, S. Chiesa, R. T. Scalettar, and G. G. Batrouni, Phys. Rev. B 87, 125141 (2013).

[59] D. Duffy and A. Moreo, Phys. Rev. B 55, R676 (1997). 